\title{
Inter- and intramolecular-bridge-mediated electron transfer in aliphatic halides.
}

\author{
Jorge G. Uranga, D. Mariano A. Vera, Ana N. Santiago and A. B. Pierini*
}

INFIQC. Departamento de Química Orgánica - Facultad de Ciencias Químicas - Universidad Nacional de Córdoba.

Ciudad Universitaria - 5000 Córdoba, Argentina

\section{Supporting Info}

\section{Table of Contents}

PART1. XYZ COORDINATES OF THE SOLUTION PHASE STRUCTURES ...................................... 4

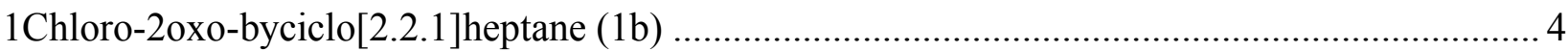

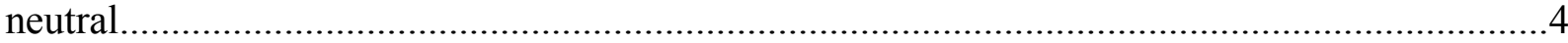

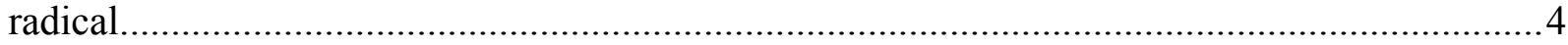

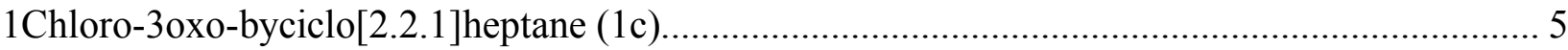

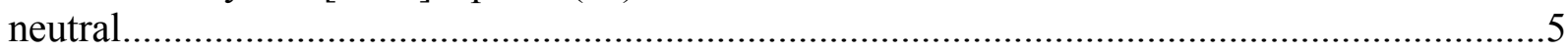

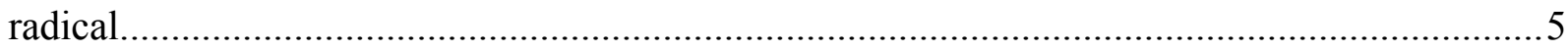

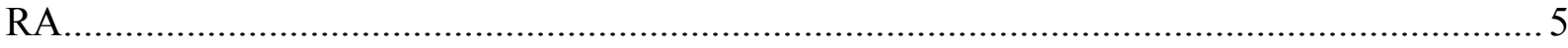

TS

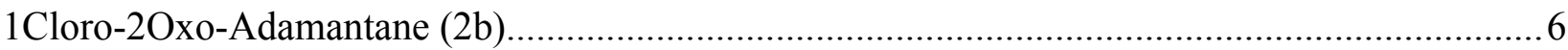

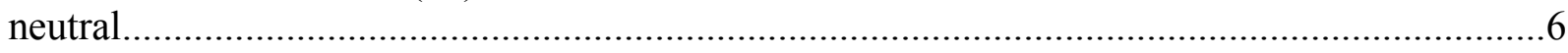

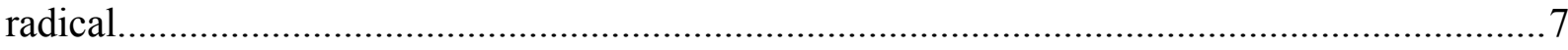

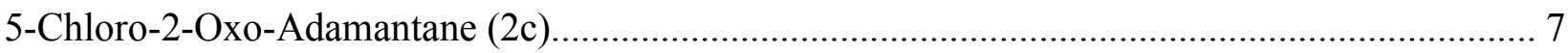

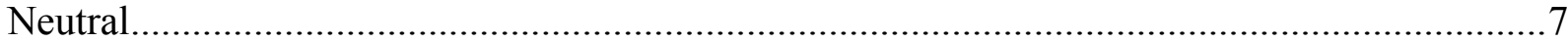

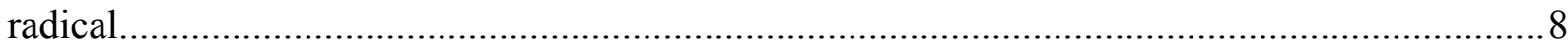

RA.

TS.

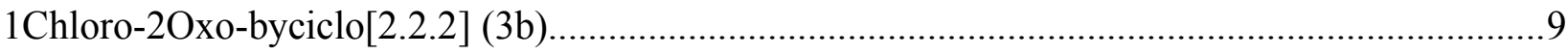

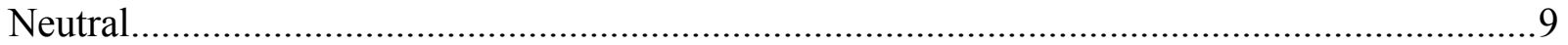




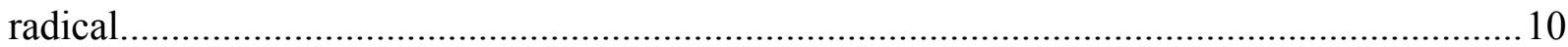

RA …

TS

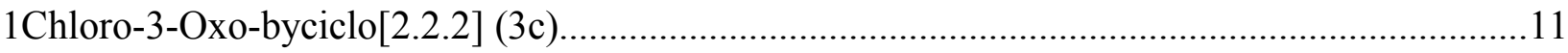

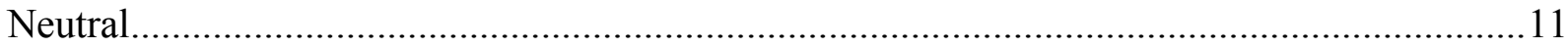

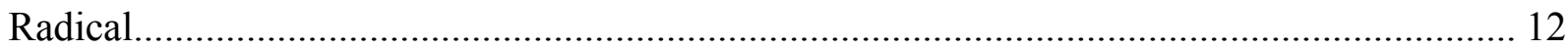

RA.

TS

1Chloro-byciclo[7.3.1.0]tridecan-13-one (4b)....................................................................13

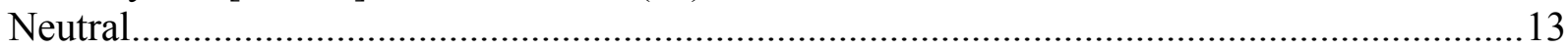

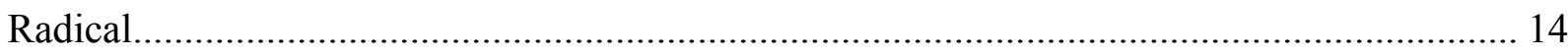

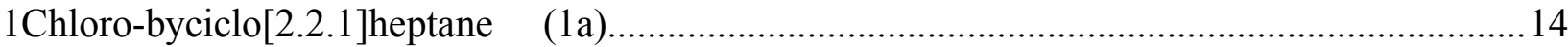

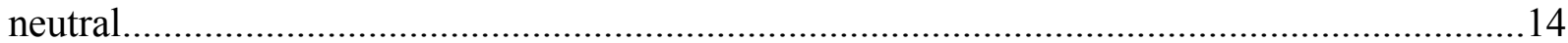

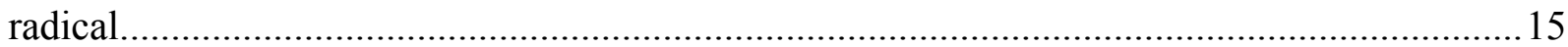

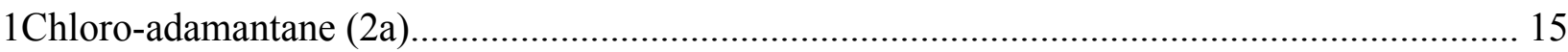

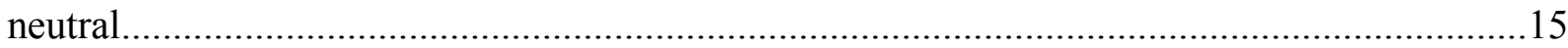

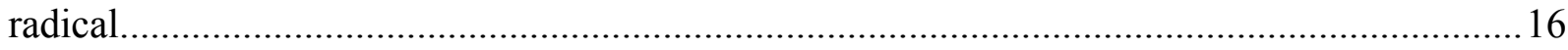

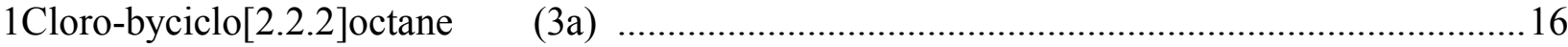

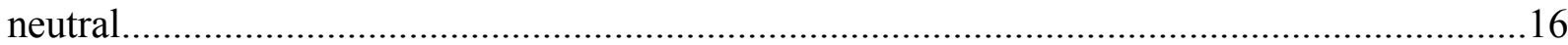

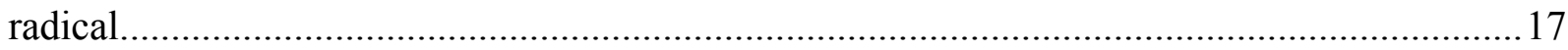

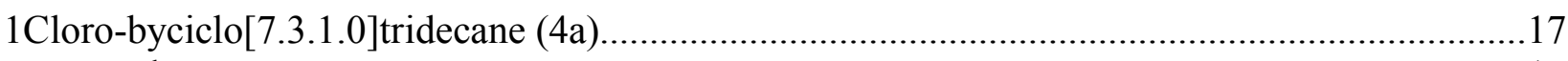

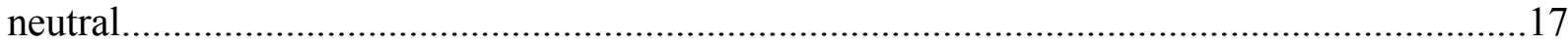

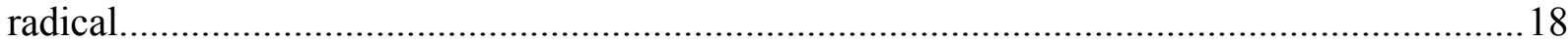

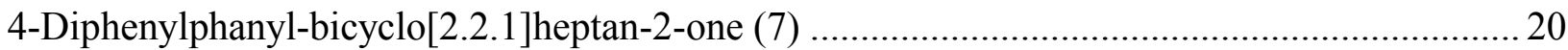

Radical Anion of 4-Diphenylphanyl-bicyclo[2.2.1] heptan-2-one (7--).................................... 20

Neutral molecule of Bicyclo[2.2.1] hept-1-yl-diphenyl-phosphane ( 8 ) .........................................2

Radical Anion of Bicyclo[2.2.1]hept-1-yl-diphenyl-phosphane ( 8-- )..........................................22

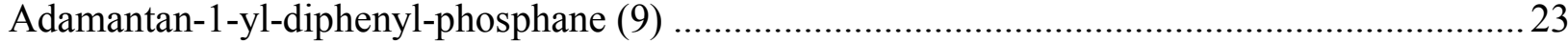

Radical Anion of Adamantan-1-yl-diphenyl-phosphane (9--) ..................................................... 24

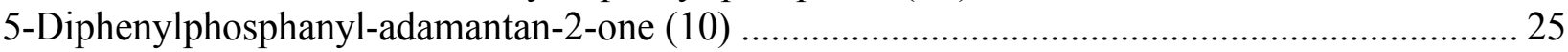

Radical Anion of 5-Diphenylphosphanyl-adamantan-2-one (10--) ............................................. 26

5-Trimehylstannyl-adamantan-2-one (11) .............................................................................. 26

Radical Anion 5-Trimehylstannyl-adamantan-2-one (11--) .........................................................27

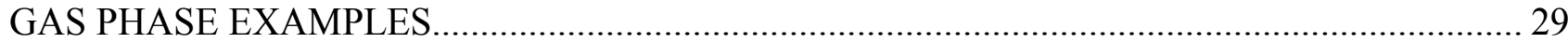

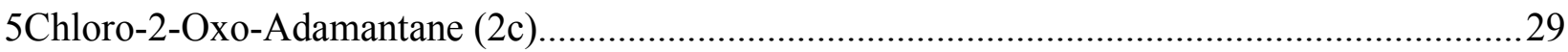

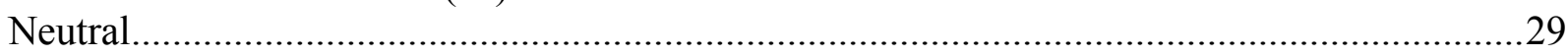

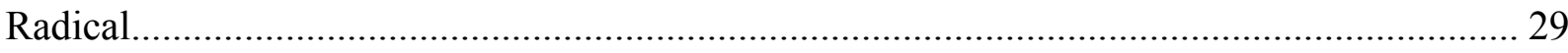

RA.

TS

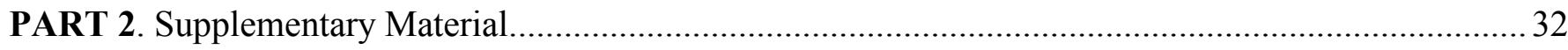

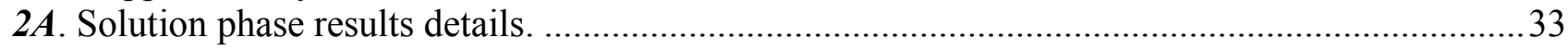

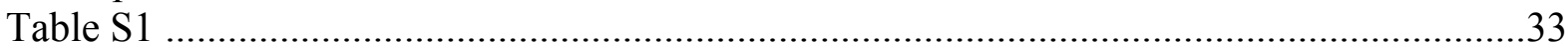

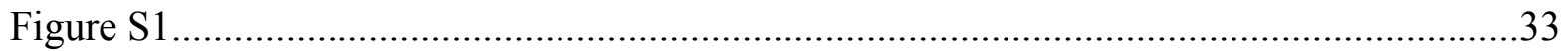

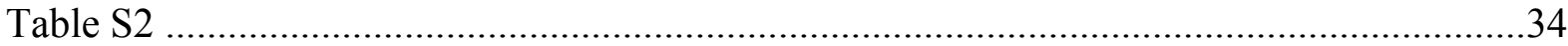

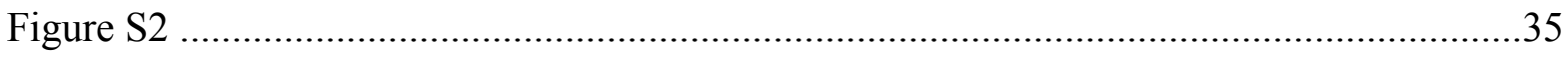


2B.Gas phase results obtained with different DFT methods.

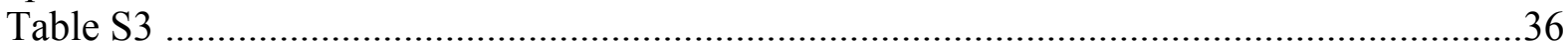

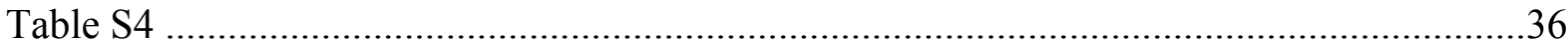

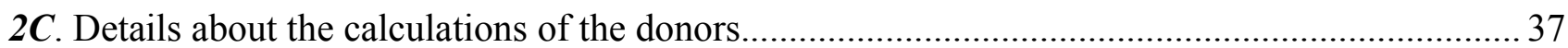

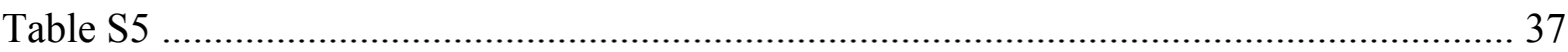

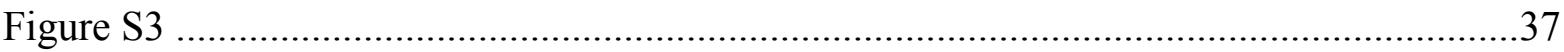

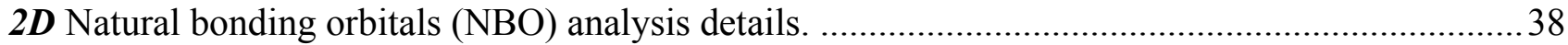

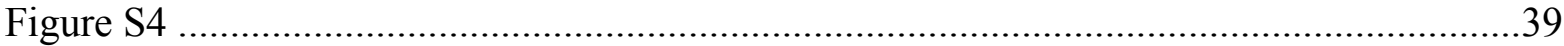




\section{PART1. XYZ COORDINATES OF THE SOLUTION PHASE STRUCTURES}

\section{coordinates in Angstrom, energies in Hartree}

\section{Chloro-2oxo-byciclo[2.2.1]heptane (1b)}

\section{neutral}

UBPW91/6-31+G* -807.5577887958

xyz coordinates:

$\begin{array}{lr}\mathrm{C} & -0.5965523238 \\ \mathrm{C} & -0.1087340861 \\ \mathrm{C} & -0.1023951205 \\ \mathrm{C} & 0.7848420728 \\ \mathrm{C} & -0.0513758444 \\ \mathrm{C} & 2.0266729529 \\ \mathrm{C} & 1.4141199398 \\ \mathrm{C} I & -1.0589218147 \\ \mathrm{O} & -1.2641237579 \\ \mathrm{H} & -1.1086262430 \\ \mathrm{H} & 1.0174744692 \\ \mathrm{H} & 0.3684835529 \\ \mathrm{H} & -0.9101409550 \\ \mathrm{H} & 2.5690722994 \\ \mathrm{H} & 1.7739609320 \\ \mathrm{H} & 1.6089936950 \\ \mathrm{H} & 2.7316755663 \\ \mathrm{H} & 0.5018627272 \\ \mathrm{H} & -10106\end{array}$

$$
\begin{array}{rr}
0.9736319911 & 0.4190734668 \\
-0.4522111110 & 0.1143031581 \\
-0.4554283563 & -1.4321760412 \\
0.8143194474 & -1.5315083333 \\
1.8334280425 & -0.7167787669 \\
0.4094668522 & -0.6874327152 \\
-0.4598326956 & 0.4600669571 \\
-1.7353847348 & 0.9463813624 \\
1.3364790414 & 1.3820221029 \\
-0.3299040874 & -1.8651576594 \\
1.1475562715 & -2.5524825290 \\
-1.3620870190 & -1.8444313279 \\
2.2168527994 & -1.3004230054 \\
1.2883962867 & -0.3036602959 \\
-1.5009681299 & 0.4271448418 \\
-0.0683352131 & 1.4707588676 \\
-0.1828292999 & -1.2920871087 \\
2.7051395514 & -0.3273161313
\end{array}
$$

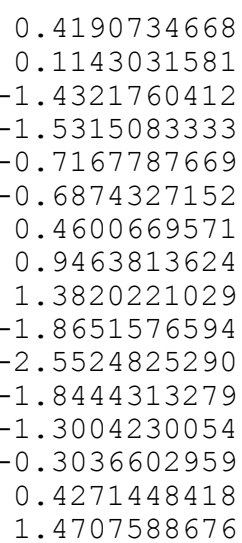

\section{radical}

UBPW91/6-31+G*, -347.28088502352

xyz coordinates:

$\begin{array}{lr}\mathrm{C} & -0.4787854360 \\ \mathrm{C} & 0.0254756883 \\ \mathrm{C} & -0.0098481552 \\ \mathrm{C} & 0.8747925023 \\ \mathrm{C} & 0.0082239352 \\ \mathrm{C} & 2.1218853623 \\ \mathrm{C} & 1.5109311808 \\ \mathrm{O} & -1.0672858814 \\ \mathrm{H} & -1.0144427721 \\ \mathrm{H} & 1.1127601360 \\ \mathrm{H} & 0.4670210530 \\ \mathrm{H} & -0.8635373986 \\ \mathrm{H} & 2.6485885082 \\ \mathrm{H} & 1.8763091459 \\ \mathrm{H} & 1.7352603836 \\ \mathrm{H} & 2.8395948758 \\ \mathrm{H} & 0.5362672620\end{array}$

$$
\begin{array}{rr}
1.0801607721 & 0.4197518627 \\
-0.2966062238 & 0.1034700078 \\
-0.3717801822 & -1.4241375012 \\
0.9101542170 & -1.5532643397 \\
1.9344436750 & -0.7656945420 \\
0.5194396540 & -0.6969853646 \\
-0.3711277871 & 0.4647822331 \\
1.4811354593 & 1.4263586419 \\
-0.2517810835 & -1.8616832233 \\
1.2261330604 & -2.5803907910 \\
-1.2787739239 & -1.8285982653 \\
2.2681657965 & -1.3608149618 \\
1.4036891560 & -0.3023544253 \\
-1.4105152361 & 0.4091657168 \\
0.0171578712 & 1.4701225921 \\
-0.0622254733 & -1.2974683352 \\
2.8343179304 & -0.4065523392
\end{array}
$$




\section{Chloro-3oxo-byciclo[2.2.1]heptane (1c)}

\section{neutral}

UBPW91/6-31+G*, -807.56094802736

xyz coordinates:

$\begin{array}{lrrr}\mathrm{C} & 0.0434741937 & 0.0152815586 & 0.0200260524 \\ \mathrm{C} & 0.0663476833 & -0.0391546983 & 1.5642432876 \\ \mathrm{C} & 1.5766768831 & -0.0826162232 & 1.8947990763 \\ \mathrm{C} & 2.1703479638 & 0.8615586167 & 0.7981828394 \\ \mathrm{C} & 0.9160416624 & 1.2967081328 & -0.0471210365 \\ \mathrm{C} & 0.1437502216 & 2.2268742437 & 0.8733557035 \\ \mathrm{C} & -0.5044357670 & 1.3415647997 & 1.9480811458 \\ \mathrm{C} 1 & -0.8717365877 & -1.4178663387 & 2.3009953686 \\ \mathrm{H} & -1.6020069629 & 1.3765732887 & 1.8183170015 \\ \mathrm{H} & -0.9705728078 & 0.1486300887 & -0.3908709127 \\ \mathrm{H} & 1.1526692466 & 1.7200447889 & -1.0319197095 \\ \mathrm{H} & 0.5236011903 & -0.8564543365 & -0.4523858962 \\ \mathrm{H} & 2.7121706525 & 1.7246106478 & 1.2153107215 \\ \mathrm{H} & 1.9643526263 & -1.1081576961 & 1.7952512670 \\ \mathrm{H} & 1.7793248434 & 0.2611337810 & 2.9214467220 \\ \mathrm{H} & 2.8633652955 & 0.3184066013 & 0.1360159869 \\ \mathrm{H} & -0.2675054290 & 1.6818126872 & 2.9693474443 \\ \mathrm{O} & 0.0605099907 & 3.4526213998 & 0.8034297232\end{array}$

\section{radical}

UBPW91/6-31+G*, -347.28557656694

xyz coordinates:

$\begin{array}{lr}\mathrm{C} & 0.0051157618 \\ \mathrm{C} & 0.0240192045 \\ \mathrm{C} & 1.4990827799 \\ \mathrm{C} & 2.1095378394 \\ \mathrm{C} & 0.8596384120 \\ \mathrm{C} & 0.0364442108 \\ \mathrm{C} & -0.6344522671 \\ \mathrm{H} & -1.7264998167 \\ \mathrm{H} & -0.9975880784 \\ \mathrm{H} & 1.1081986873 \\ \mathrm{H} & 0.5230469244 \\ \mathrm{H} & 2.6388650581 \\ \mathrm{H} & 1.9258569350 \\ \mathrm{H} & 1.6858744201 \\ \mathrm{H} & 2.8149716932 \\ \mathrm{H} & -0.4531466594 \\ \mathrm{O} & -0.0502395987\end{array}$
0.0068678845
0.0164762059
$-0.0186057454$
0.9167172444
1.3221110421
2. 2430945121
1.3362682903
1.3416604087
0.1197919730
1.7425502595
$-0.8468117053$
1.7918779528
$-1.0332700961$
0.3646259852
0.3654878361
1.7238983263
$-0.0063114408$
1.5195443910
1.9028103926
0.7831012499
$-0.0853989694$
0.8033540171
1.8760446416
1.6937959415
$-0.4504949924$
$-1.0706311583$
$-0.4736352028$
1.1922654968
1.8393282879
2.9188379473
0.1396943986
2.8924005072
3.4678539460
0.7363326042

\section{RA}

UBPW91/6-31+G*, -807.63029644619

xyz coordinates:

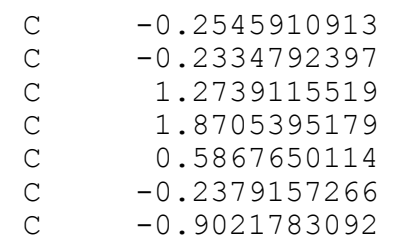

-0.6001518270
-0.5230663989
-0.4931296014
0.3094003045
0.6942436957
1.6609308876
0.8064222206

-1.2566210170
0.2759269933
0.6172589047
-0.5917063852
-1.3900080168
-0.5321101340
0.5837585365 


$\begin{array}{lrrr}\mathrm{Cl} & -1.1080152472 & -2.0339764930 & 1.1229366103 \\ \mathrm{H} & -2.0068427429 & 0.7522800610 & 0.5258326673 \\ \mathrm{H} & -1.2680241873 & -0.4986893877 & -1.6764695356 \\ \mathrm{H} & 0.7964405120 & 1.0538782725 & -2.4128286471 \\ \mathrm{H} & 0.2357718512 & -1.4993533607 & -1.6620318906 \\ \mathrm{H} & 2.4291267373 & 1.2029076199 & -0.2680408720 \\ \mathrm{H} & 1.6804519788 & -1.5162959046 & 0.6662013406 \\ \mathrm{H} & 1.4550664179 & -0.0137765120 & 1.5937802527 \\ \mathrm{H} & 2.5596517928 & -0.3112119749 & -1.1945582328 \\ \mathrm{H} & -0.6477076329 & 1.1864852721 & 1.5915912761 \\ \mathrm{O} & 0.1349416297 & 2.8991373973 & -0.3172260335 \\ -\end{array}$

\section{TS}

UBPW91/6-31+G*, $-807.628384078354,1$ imaginary freq. xyz coordinates:

$\begin{array}{rrrr}\mathrm{C} & -0.924558 & 0.809858 & 0.576217 \\ \mathrm{C} & -0.236588 & -0.505136 & 0.271009 \\ \mathrm{C} & -0.267049 & -0.591230 & -1.259449 \\ \mathrm{C} & 0.578794 & 0.699866 & -1.399426 \\ \mathrm{C} & -0.218104 & 1.667805 & -0.511530 \\ \mathrm{C} & 1.868428 & 0.311350 & -0.612232 \\ \mathrm{C} & 1.268575 & -0.470367 & 0.608988 \\ \mathrm{Cl} & -1.106457 & -2.063147 & 1.131180 \\ \mathrm{O} & 0.180614 & 2.885493 & -0.279650 \\ \mathrm{H} & -2.028001 & 0.745479 & 0.470993 \\ \mathrm{H} & -1.283142 & -0.495504 & -1.675197 \\ \mathrm{H} & 0.778946 & 1.070378 & -2.421303 \\ \mathrm{H} & 0.222971 & -1.490789 & -1.665349 \\ \mathrm{H} & 2.437521 & 1.202473 & -0.301725 \\ \mathrm{H} & 1.677825 & -1.490804 & 0.676308 \\ \mathrm{H} & 1.451372 & 0.027067 & 1.575567 \\ \mathrm{H} & 2.546516 & -0.322739 & -1.214676 \\ \mathrm{H} & -0.707793 & 1.193164 & 1.590028\end{array}$

\section{Cloro-2Oxo-Adamantane (2b)}

\section{neutral}

UBPW91/6-31+G*, -924.29611144316

xyz coordinates:

$\begin{array}{lr}\mathrm{C} & 0.0129416492 \\ \mathrm{C} & 0.0039387724 \\ \mathrm{C} & 1.4650864486 \\ \mathrm{C} & 2.2180562809 \\ \mathrm{C} & 2.2283758857 \\ \mathrm{C} & 0.7769752249 \\ \mathrm{C} & 1.5118240682 \\ \mathrm{C} & 0.0629028394 \\ \mathrm{C} & -0.6670436315 \\ \mathrm{C} & 0.0578418268 \\ \mathrm{O} & -1.6712795813 \\ \mathrm{H} & 2.0368515395 \\ \mathrm{H} & 3.2514562212 \\ \mathrm{H} & 2.7536888313 \\ \mathrm{H} & 2.7800171713\end{array}$

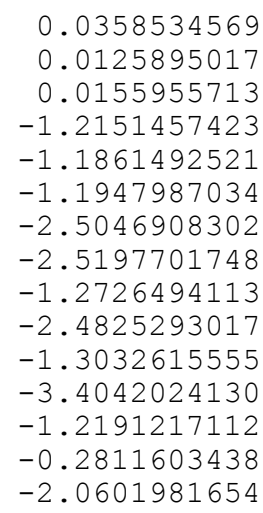

$-0.0502496532$

1.5054353779

2.0415427090

1.4952673035

$-0.0498992306$

$-0.5803935310$

1.9854367342

1. 4413456101

1.9646856207

$-0.1066394461$

2. 6759876155

1.6191221187

1.8836829983

$-0.4055478550$

$-0.4428195961$ 


$\begin{array}{lrrr}\mathrm{H} & 0.7747271433 & -1.1853689022 & -1.6842325999 \\ \mathrm{H} & -0.9816371085 & -2.5091367934 & -0.4783970534 \\ \mathrm{H} & 0.5733025287 & -3.3834102726 & -0.4832145971 \\ \mathrm{H} & -1.0232609377 & 0.0445154127 & -0.4338746996 \\ \mathrm{H} & 0.4937709985 & 0.9718360703 & -0.3876282316 \\ \mathrm{H} & 1.9572648461 & 0.9514732333 & 1.7203954532 \\ \mathrm{H} & 1.4619465946 & 0.0107072925 & 3.1464414668 \\ \mathrm{H} & -0.5699105723 & 0.8601776737 & 1.9132294757 \\ \mathrm{H} & 1.4986919631 & -2.5480109253 & 3.0886512523 \\ \mathrm{Cl} & -0.7859243618 & -4.0482647789 & 2.0158335513 \\ -\end{array}$

\section{radical}

UBPW91/6-31+G*, -464.02882712586

xyz coordinates:

$\begin{array}{lrrr}\mathrm{C} & -1.3657806866 & 0.1415012074 & -0.4020060106 \\ \mathrm{C} & -1.3510334184 & 0.1347315731 & 1.1564457103 \\ \mathrm{C} & 0.1037740329 & 0.1451848411 & 1.6765336434 \\ \mathrm{C} & 0.8345351788 & -1.1425277106 & 1.1603463645 \\ \mathrm{C} & 0.8025556620 & -1.0573185277 & -0.3530947815 \\ \mathrm{C} & -0.5976572445 & -1.0930403991 & -0.8744192281 \\ \mathrm{C} & 1.5772649057 & 0.1160920460 & -0.9205559266 \\ \mathrm{C} & 0.8455757989 & 1.4023159824 & -0.4018875322 \\ \mathrm{C} & 0.8461768479 & 1.3932614517 & 1.1446008941 \\ \mathrm{C} & -0.6068054499 & 1.3963911174 & -0.9290746541 \\ \mathrm{O} & -1.0897697639 & -1.9800131226 & -1.5860149283 \\ \mathrm{H} & 1.8753572776 & -1.1681217910 & 1.5311306019 \\ \mathrm{H} & 0.1051647148 & 0.1400271742 & 2.7813471090 \\ \mathrm{H} & 0.3533191743 & 2.3083441138 & 1.5218529721 \\ \mathrm{H} & 1.8850029374 & 1.4058233871 & 1.5231534616 \\ \mathrm{H} & 1.3763991399 & 2.2943506233 & -0.7803448755 \\ \mathrm{H} & 1.5887544405 & 0.1092860774 & -2.0256056404 \\ \mathrm{H} & 2.6252483886 & 0.1023782370 & -0.5697965565 \\ \mathrm{H} & -0.6185475279 & 1.4079983870 & -2.0341351277 \\ \mathrm{H} & -1.1442005047 & 2.3012172976 & -0.5907734128 \\ \mathrm{H} & -1.8969785842 & 1.0252781003 & 1.5180365898 \\ \mathrm{H} & -1.8919459396 & -0.7515868668 & 1.5350846755 \\ \mathrm{H} & -2.3963118362 & 0.1030907645 & -0.7932445162 \\ \mathrm{H} & 0.3178390966 & -2.0445819803 & 1.5352745995\end{array}$

\section{5-Chloro-2-Oxo-Adamantane (2c)}

\section{Neutral}

UBPW91/6-31+G*, -924.302018994

xyz coordinates:

$\begin{array}{lrrr}\text { C } & 0.000842 & -0.001735 & -0.001683 \\ \text { C } & 0.004879 & -0.008940 & 1.535445 \\ \text { C } & 1.444970 & -0.000790 & 2.076157 \\ \text { C } & 2.169215 & -1.283445 & 1.570618 \\ \text { C } & 2.169386 & -1.306006 & 0.014719 \\ \text { C } & 0.714973 & -1.285245 & -0.503297 \\ \text { C } & 1.387843 & -2.495487 & 2.054499 \\ \text { C } & -0.053915 & -2.520032 & 1.570386 \\ \text { C } & -0.761595 & -1.228184 & 2.076144 \\ \text { C } & -0.034584 & -2.531827 & 0.014479\end{array}$




\begin{tabular}{|c|c|c|c|}
\hline $\mathrm{Cl}$ & -0.850714 & 1.529469 & 2.143519 \\
\hline $\mathrm{H}$ & 3.194208 & -1.322871 & 1.971909 \\
\hline $\mathrm{H}$ & 2.732455 & -0.428660 & -0.352648 \\
\hline $\mathrm{H}$ & 2.694485 & -2.207465 & -0.348434 \\
\hline $\mathrm{H}$ & 0.714721 & -1.284971 & -1.606878 \\
\hline $\mathrm{H}$ & -1.076905 & -2.547373 & -0.353063 \\
\hline $\mathrm{H}$ & 0.454479 & -3.453281 & -0.348845 \\
\hline $\mathrm{H}$ & -0.561014 & -3.411860 & 1.971278 \\
\hline $\mathrm{H}$ & 1.441425 & 0.029352 & 3.179743 \\
\hline $\mathrm{H}$ & 1.990491 & 0.889436 & 1.717481 \\
\hline $\mathrm{H}$ & -0.785335 & -1.209269 & 3.179723 \\
\hline $\mathrm{H}$ & -1.805666 & -1.222060 & 1.717598 \\
\hline $\mathrm{H}$ & 0.521514 & 0.897541 & -0.375073 \\
\hline $\mathrm{H}$ & -1.037785 & 0.029897 & -0.375121 \\
\hline 0 & 1.881973 & -3.383870 & 2.760512 \\
\hline
\end{tabular}

\section{radical}

UBPW91/6-31+G*, -464.03252250414

xyz coordinates:

$\begin{array}{cr}\mathrm{C} & -0.0631981409 \\ \mathrm{C} & -0.0338564785 \\ \mathrm{C} & 1.4612663311 \\ \mathrm{C} & 2.0231674117 \\ \mathrm{C} & 2.1067073567 \\ \mathrm{C} & 0.6058427774 \\ \mathrm{C} & -0.7844484584 \\ \mathrm{C} & -0.1206920082 \\ \mathrm{C} & 1.3906968323 \\ \mathrm{C} & -0.1058238709 \\ \mathrm{O} & -0.6056144449 \\ \mathrm{H} & -0.6594597820 \\ \mathrm{H} & -0.7742158320 \\ \mathrm{H} & -1.8442954033 \\ \mathrm{H} & -0.5113582943 \\ \mathrm{H} & 0.4710046723 \\ \mathrm{H} & -1.1057794126 \\ \mathrm{H} & 0.5673668586 \\ \mathrm{H} & 1.8720937188 \\ \mathrm{H} & 1.3953530329 \\ \mathrm{H} & 2.5990865258 \\ \mathrm{H} & 2.6351062302 \\ \mathrm{H} & 1.4897756972 \\ \mathrm{H} & 1.9993913349 \\ ----------------1\end{array}$

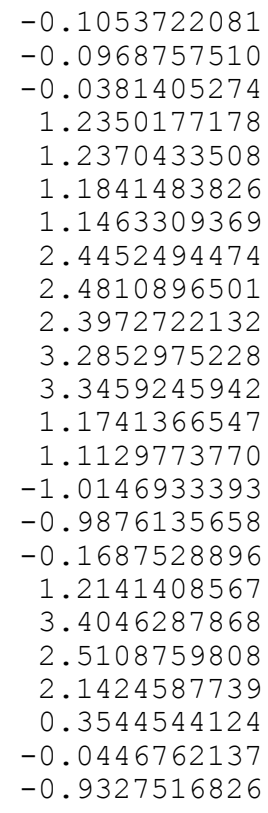

0.0413358695

1.5856396672

2.0701793024

1.4955341552

$-0.0057072323$

$-0.5174248795$

2.1117582567

1.5693688052

2.0508273910

0.0528771987

$-0.6575572034$

1. 9079337347

3. 2172413017

1.7995700122

1.9740430310

$-0.3579406299$

$-0.3206126684$

$-1.6189571511$

1.6816101206

3. 1552671859

$-0.4041225918$

$-0.4088671538$

3.1752704208

1.7066533715

\section{RA}

UBPW91/6-31+G*, $\quad-924.36750319736$

xyz coordinates:

\footnotetext{
C $\quad-1.4535480923$

C $\quad-0.1133165519$

C $\quad 1.0437600060$

C $\quad 1.0713865098$

C $\quad-0.2728326585$

C $\quad-1.4284214172$

C $\quad 1.2338494645$

C $\quad 0.1208198486$

C $\quad 0.0898958529$

C $\quad-1.2308810246$
}

$-0.0096982543$

0.7350327250

$-0.2230093880$

$-1.2931925945$

$-2.0744698167$

$-1.0861786516$

$-0.6309322904$

0.3817845455

1.4572558768

$-0.3863071163$
-0.8375569209
-0.8217497429
-1.1207355713
0.0019362080
0.0040526961
0.2831541552
1.3688303053
1.6309058806
0.5135871605
1.6474986496 


\begin{tabular}{|c|c|c|c|}
\hline $\mathrm{Cl}$ & -0.1560433115 & 2.0486220232 & -2.1975696718 \\
\hline $\mathrm{H}$ & -0.4394979427 & -2.5958327525 & -0.9601648619 \\
\hline $\mathrm{H}$ & -0.2361365217 & -2.8485100923 & 0.7909169964 \\
\hline $\mathrm{H}$ & -2.3922511796 & -1.6255141088 & 0.2746678153 \\
\hline $\mathrm{H}$ & -1.2066559536 & -1.1354290613 & 2.4587173743 \\
\hline $\mathrm{H}$ & 0.2832457562 & 0.8800554625 & 2.6044053926 \\
\hline $\mathrm{H}$ & 2.0008445100 & 0.3264732592 & -1.1374035799 \\
\hline $\mathrm{H}$ & -0.7297082440 & 2.1780881252 & 0.6855460747 \\
\hline $\mathrm{H}$ & -1.6099936720 & -0.4875168315 & -1.8206811194 \\
\hline $\mathrm{H}$ & -2.2827159465 & 0.6988979634 & -0.6656080777 \\
\hline O & 1.6293415994 & -1.3942832755 & 2.3849991597 \\
\hline
\end{tabular}

\section{TS}

UBPW91/6-31+G*,-924.362802075953, 1 imaginary freq. xyz coordinates:

$\begin{array}{rrrr}\mathrm{C} & -1.430714 & 0.027526 & -0.866685 \\ \mathrm{C} & -0.091591 & 0.754318 & -0.833651 \\ \mathrm{C} & 1.060614 & -0.190517 & -1.144662 \\ \mathrm{C} & 1.086670 & -1.269569 & -0.017557 \\ \mathrm{C} & -0.261595 & -2.046778 & -0.023058 \\ \mathrm{C} & -1.414225 & -1.054893 & 0.254509 \\ \mathrm{C} & 1.243005 & -0.605662 & 1.342151 \\ \mathrm{C} & 0.137446 & 0.404374 & 1.609931 \\ \mathrm{C} & 0.108824 & 1.486490 & 0.485544 \\ \mathrm{C} & -1.219862 & -0.356980 & 1.620049 \\ \mathrm{Cl} & -0.128745 & 2.137312 & -2.276917 \\ \mathrm{H} & 1.926704 & -1.965932 & -0.198429 \\ \mathrm{H} & -0.425483 & -2.564421 & -0.990496 \\ \mathrm{H} & -0.231909 & -2.825167 & 0.760378 \\ \mathrm{H} & -2.381296 & -1.588566 & 0.239211 \\ \mathrm{H} & -2.066973 & 0.329881 & 1.823555 \\ \mathrm{H} & -1.206970 & -1.105873 & 2.432211 \\ \mathrm{H} & 0.302832 & 0.897020 & 2.586261 \\ \mathrm{H} & 2.020587 & 0.352474 & -1.162432 \\ \mathrm{H} & 0.916715 & -0.667495 & -2.130342 \\ \mathrm{H} & 1.058699 & 2.047196 & 0.483750 \\ \mathrm{H} & -0.713169 & 2.202757 & 0.661750 \\ \mathrm{H} & -1.587735 & -0.449397 & -1.850079 \\ \mathrm{H} & -2.259304 & 0.736395 & -0.693065 \\ \mathrm{O} & 1.744947 & -1.299542 & 2.348400\end{array}$

\section{Chloro-2Oxo-byciclo[2.2.2] (3b)}

\section{Neutral}

UBPW91/6-31+G*, $\quad-846.8716525021$

xyz coordinates:
C $\quad-0.0223070432$
C $\quad-0.0181197528$
$-0.0056631013$
0.0006503234
$-0.0001557170$
1.5461478135
C $\quad 1.4517113859$
$-0.0194769562$
2.0754555867 


\begin{tabular}{|c|c|c|c|}
\hline $\mathrm{C}$ & 2.4343438541 & -0.0673775993 & 0.8775004315 \\
\hline $\mathrm{C}$ & 2.1914204258 & 1.1771242870 & 0.0180805821 \\
\hline $\mathrm{C}$ & 0.7594545998 & 1.2251935447 & -0.4963177472 \\
\hline $\mathrm{C}$ & 0.6866801619 & -1.2783973540 & -0.5158242431 \\
\hline $\mathrm{C}$ & 2.1577389325 & -1.3199469321 & 0.0086736176 \\
\hline $\mathrm{Cl}$ & 4.1545685719 & -0.1098332637 & 1.4924129375 \\
\hline $\mathrm{H}$ & 0.3100606864 & 2.1757683186 & -0.1517019097 \\
\hline $\mathrm{H}$ & 1.6713765695 & 0.8659454685 & 2.6934696501 \\
\hline $\mathrm{H}$ & 1.6439713815 & -0.9064345871 & 2.7015293772 \\
\hline 0 & 3.0319551265 & 2.0398953316 & -0.2300835996 \\
\hline $\mathrm{H}$ & 2.8822821390 & -1.3583461313 & -0.8204979214 \\
\hline $\mathrm{H}$ & 2.3374126859 & -2.2080371077 & 0.6368192645 \\
\hline $\mathrm{H}$ & 0.6782350926 & -1.2869088224 & -1.6190901319 \\
\hline $\mathrm{H}$ & 0.1369842298 & -2.1736218734 & -0.1806718815 \\
\hline $\mathrm{H}$ & -0.5709584356 & -0.8769684742 & 1.9223770049 \\
\hline $\mathrm{H}$ & -0.5417323703 & 0.8971652143 & 1.9173346831 \\
\hline $\mathrm{H}$ & -1.0579387573 & 0.0287323061 & -0.3749233089 \\
\hline $\mathrm{H}$ & 0.7995940602 & 1.2787706844 & -1.6006129486 \\
\hline
\end{tabular}

\section{radical}

UBPW91/6-31+G*, $\quad-386.60194673218$

xyz coordinates:

\begin{tabular}{|c|c|c|c|}
\hline $\mathrm{C}$ & 0.0000000000 & 0.0000000000 & 0.0000000000 \\
\hline $\mathrm{C}$ & 0.0000000000 & 0.0000000000 & 1.5495690000 \\
\hline $\mathrm{C}$ & 1.4939850000 & 0.0000000000 & 2.0759440000 \\
\hline $\mathrm{C}$ & 2.3900510000 & -0.0190800000 & 0.8522650000 \\
\hline $\mathrm{C}$ & 2.1951720000 & 1.2102570000 & 0.0298980000 \\
\hline $\mathrm{C}$ & 0.7540530000 & 1.2543630000 & -0.4990220000 \\
\hline $\mathrm{C}$ & 0.7298170000 & -1.2628720000 & -0.5234200000 \\
\hline $\mathrm{C}$ & 2.2212190000 & -1.2689590000 & 0.0104100000 \\
\hline $\mathrm{H}$ & 0.2841740000 & 2.1931230000 & -0.1485930000 \\
\hline $\mathrm{H}$ & 1.6838750000 & 0.8855010000 & 2.7047420000 \\
\hline $\mathrm{H}$ & 1.6906740000 & -0.8915110000 & 2.6955070000 \\
\hline 0 & 3.0371290000 & 2.0837960000 & -0.2124770000 \\
\hline $\mathrm{H}$ & 2.9343400000 & -1.2927920000 & -0.8301140000 \\
\hline $\mathrm{H}$ & 2.4120350000 & -2.1626520000 & 0.6287260000 \\
\hline $\mathrm{H}$ & 0.7237980000 & -1.2700730000 & -1.6272010000 \\
\hline $\mathrm{H}$ & 0.2001380000 & -2.1699840000 & -0.1866440000 \\
\hline $\mathrm{H}$ & -0.5372600000 & -0.8871540000 & 1.9252160000 \\
\hline $\mathrm{H}$ & -0.5332170000 & 0.8902330000 & 1.9257390000 \\
\hline $\mathrm{H}$ & -1.0375270000 & 0.0132090000 & -0.3732320000 \\
\hline $\mathrm{H}$ & 0.7906510000 & 1.3102360000 & -1.6036540000 \\
\hline
\end{tabular}

\section{RA}

UBPW91/6-31+G*, -846.93448283745

xyz coordinates:

$\begin{array}{lr}\text { C } & -0.0122507011 \\ \mathrm{C} & -0.0117441407 \\ \mathrm{C} & 1.4490565803 \\ \mathrm{C} & 2.4142602114 \\ \mathrm{C} & 2.1629249765 \\ \mathrm{C} & 0.6957739853 \\ \mathrm{C} & 0.7802861218 \\ \mathrm{C} & 2.1902988952 \\ \mathrm{C} 1 & 4.1618239177 \\ \mathrm{H} & 0.1867625758 \\ \mathrm{H} & 1.5926522110 \\ \mathrm{H} & 1.6972553053\end{array}$

0.0164706559
-0.0548814870
0.0957012696
0.0368792333
1.2053810054
1.3248261975
-1.1844713879
-1.2868344108
0.0502045058
2.2001585288
1.0602814669
-0.7007910075
0.0205523716
1.5632426630
2.0881476076
0.8749066857
$-0.0640085730$
$-0.4033387178$
$-0.5549568858$
0.1180787863
1. 5466025600
0.0894335059
2. 6024960758
2.8113457413 


$\begin{array}{lrrr}\mathrm{O} & 3.0569372412 & 2.0968867546 & -0.4476240850 \\ \mathrm{H} & 2.9764320979 & -1.4189772252 & -0.6424905630 \\ \mathrm{H} & 2.2450137850 & -2.1488115804 & 0.8125864978 \\ \mathrm{H} & 0.8924675562 & -1.0488580712 & -1.6450101057 \\ \mathrm{H} & 0.2173626188 & -2.1225464463 & -0.4018604047 \\ \mathrm{H} & -0.4450404119 & -1.0141502901 & 1.8963732511 \\ \mathrm{H} & -0.6510239797 & 0.7474766222 & 1.9727853072 \\ \mathrm{H} & -1.0502029474 & -0.0048742799 & -0.3565903567 \\ \mathrm{H} & 0.5706978079 & 1.4993781502 & -1.4950173749\end{array}$

\section{TS}

UBPW91/6-31+G*, -846.93349441012 , 1 imaginary freq. xyz coordinates:

$\begin{array}{lrrr}\mathrm{C} & -0.0122507011 & 0.0164706559 & 0.0205523716 \\ \mathrm{C} & -0.0117441407 & -0.0548814870 & 1.5632426630 \\ \mathrm{C} & 1.4490565803 & 0.0957012696 & 2.0881476076 \\ \mathrm{C} & 2.4142602114 & 0.0368792333 & 0.8749066857 \\ \mathrm{C} & 2.1629249765 & 1.2053810054 & -0.0640085730 \\ \mathrm{C} & 0.6957739853 & 1.3248261975 & -0.4033387178 \\ \mathrm{C} & 0.7802861218 & -1.1844713879 & -0.5549568858 \\ \mathrm{C} & 2.1902988952 & -1.2868344108 & 0.1180787863 \\ \mathrm{Cl} & 4.1618239177 & 0.0502045058 & 1.5466025600 \\ \mathrm{H} & 0.1867625758 & 2.2001585288 & 0.0894335059 \\ \mathrm{H} & 1.5926522110 & 1.0602814669 & 2.6024960758 \\ \mathrm{H} & 1.6972553053 & -0.7007910075 & 2.8113457413 \\ \mathrm{O} & 3.0569372412 & 2.0968867546 & -0.4476240850 \\ \mathrm{H} & 2.9764320979 & -1.4189772252 & -0.6424905630 \\ \mathrm{H} & 2.2450137850 & -2.1488115804 & 0.8125864978 \\ \mathrm{H} & 0.8924675562 & -1.0488580712 & -1.6450101057 \\ \mathrm{H} & 0.2173626188 & -2.1225464463 & -0.4018604047 \\ \mathrm{H} & -0.4450404119 & -1.0141502901 & 1.8963732511 \\ \mathrm{H} & -0.6510239797 & 0.7474766222 & 1.9727853072 \\ \mathrm{H} & -1.0502029474 & -0.0048742799 & -0.3565903567 \\ \mathrm{H} & 0.5706978079 & 1.4993781502 & -1.4950173749\end{array}$

\section{Chloro-3-Oxo-byciclo[2.2.2] (3c)}

\section{Neutral}

UBPW91/6-31+G*, -846.87755124254

xyz coordinates:

$\begin{array}{lr}\mathrm{C} & -0.2413979106 \\ \mathrm{C} & -0.2853675609 \\ \mathrm{C} & 1.2130508988 \\ \mathrm{C} & -0.9516468198 \\ \mathrm{H} & -1.3342620768 \\ \mathrm{C} & 0.4383515591 \\ \mathrm{H} & 0.2046189192 \\ \mathrm{H} & -0.9520178096 \\ \mathrm{C} & -0.2133856165 \\ \mathrm{H} & -2.0053730634 \\ \mathrm{H} & 1.2219416215 \\ \mathrm{H} & 1.7132601496 \\ \mathrm{C} & 1.9372064767 \\ \mathrm{H} & 2.3271329051\end{array}$
0.7525392574
0.7472289031
0.7807936369
$-0.4949794328$
0.7689176359
$-0.5414945379$
1.6559497311
$-0.5236667124$
$-1.7300849079$
$-0.5467089143$
0.8275981084
1.6890104953
$-0.5088765536$
$-1.0959369941$

\begin{abstract}
$-0.1649134560$
1. 3732624047

$-0.6666042508$

$-0.7071602183$

1.7120016757

1.8845568228

1. 7585255309

$-1.8117732237$

$-0.1843781070$

$-0.3785962054$

$-1.7680195636$

$-0.2935148085$

$-0.1583538822$

$-1.0053917858$
\end{abstract}




$\begin{array}{lrrr}\mathrm{H} & 2.8006586414 & -0.2447642523 & 0.4740782866 \\ \mathrm{C} & 0.9707620768 & -1.3831829851 & 0.6902096503 \\ \mathrm{H} & 1.2969160516 & -0.2761062260 & 2.5231152338 \\ \mathrm{H} & -0.2421781312 & -1.1526044391 & 2.4990717217 \\ \mathrm{Cl} & -1.1247097937 & 2.2616803255 & -0.7892150347 \\ \mathrm{H} & 1.4662454896 & -2.3015387283 & 1.0393088539 \\ \mathrm{O} & -0.5723674690 & -2.8767710445 & -0.4663548592\end{array}$

\section{Radical}

UBPW91/6-31+G*, $\quad-386.60695313319$

xyz coordinates:

\begin{tabular}{|c|c|c|c|}
\hline $\mathrm{C}$ & -0.00090 & 0.00650 & -0.00370 \\
\hline C & -0.01450 & -0.00470 & 1.55500 \\
\hline $\mathrm{C}$ & 1.46690 & 0.00240 & 2.11170 \\
\hline $\mathrm{C}$ & 2.37310 & 0.02920 & 0.90230 \\
\hline C & 2.19580 & 1.28660 & 0.09170 \\
\hline $\mathrm{C}$ & 0.74660 & 1.25020 & -0.45080 \\
\hline $\mathrm{C}$ & 0.78880 & -1.23410 & -0.51990 \\
\hline $\mathrm{C}$ & 2.26500 & -1.21420 & 0.04980 \\
\hline 0 & 0.27400 & 2.13970 & -1.16300 \\
\hline $\mathrm{H}$ & 1.62430 & 0.88660 & 2.75380 \\
\hline $\mathrm{H}$ & 1.64510 & -0.89330 & 2.73090 \\
\hline $\mathrm{H}$ & 2.87830 & 1.35920 & -0.77640 \\
\hline $\mathrm{H}$ & 2.31250 & 2.21780 & 0.67820 \\
\hline $\mathrm{H}$ & 2.99040 & -1.19730 & -0.78240 \\
\hline $\mathrm{H}$ & 2.45730 & -2.12690 & 0.63920 \\
\hline $\mathrm{H}$ & 0.79000 & -1.24760 & -1.62240 \\
\hline $\mathrm{H}$ & 0.25400 & -2.13940 & -0.18550 \\
\hline $\mathrm{H}$ & -0.54930 & -0.91220 & 1.88320 \\
\hline $\mathrm{H}$ & -0.58380 & 0.86100 & 1.93220 \\
\hline $\mathrm{H}$ & -1.02430 & 0.02030 & -0.40800 \\
\hline
\end{tabular}

\section{RA}

UBPW91/6-31+G*, 846.93929954591

xyz coordinates:

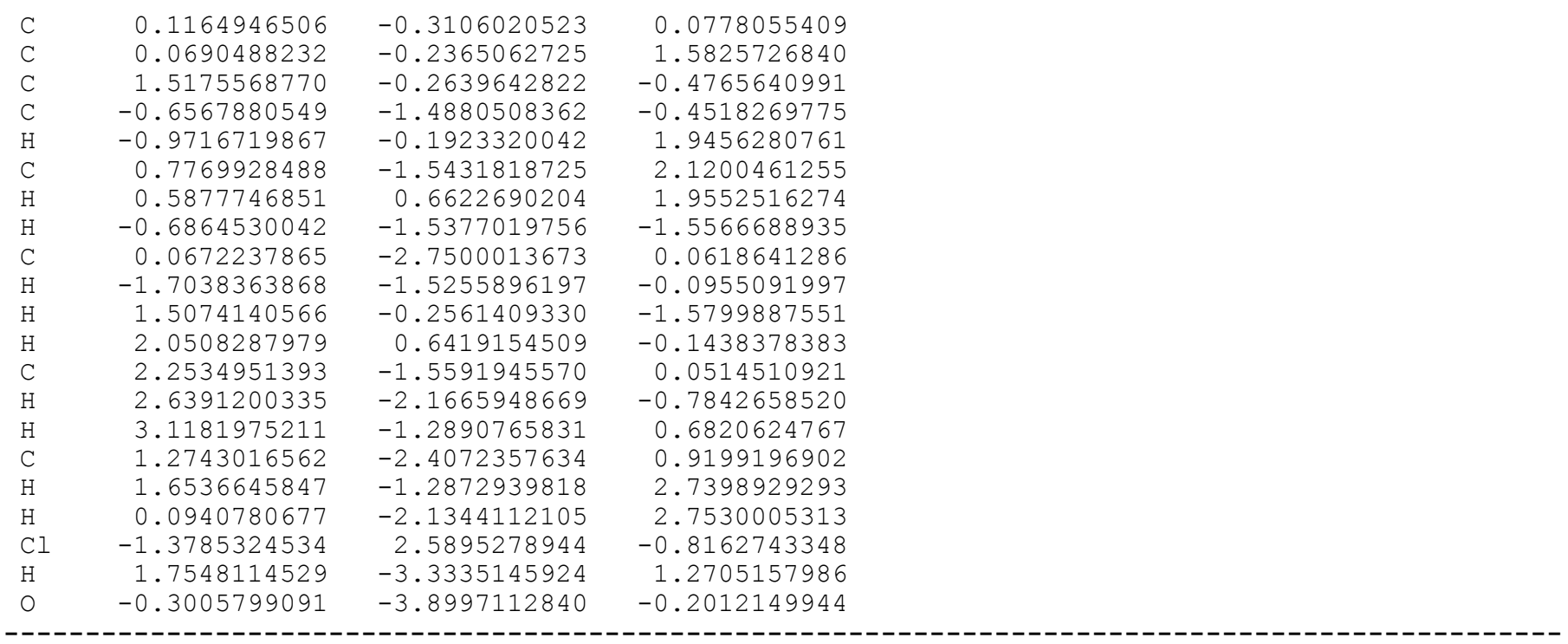




\section{TS}

UBPW91/6-31+G*, --846.93766859161, 1 imaginary freq. xyz coordinates:

$\begin{array}{lrrr}\mathrm{C} & -0.2257147147 & 0.7658246850 & -0.2051552978 \\ \mathrm{C} & -0.2893801500 & 0.7552331210 & 1.3322863462 \\ \mathrm{C} & 1.2284488438 & 0.7975273993 & -0.7070016657 \\ \mathrm{C} & -0.9489668718 & -0.4597781062 & -0.7694603823 \\ \mathrm{H} & -1.3474095130 & 0.7129539406 & 1.6424081894 \\ \mathrm{C} & 0.5033054974 & -0.4878116295 & 1.8606697596 \\ \mathrm{H} & 0.1310247822 & 1.6933429202 & 1.7308896038 \\ \mathrm{H} & -0.9472881132 & -0.4186977166 & -1.8886269892 \\ \mathrm{C} & -0.2257771683 & -1.6659761674 & -0.2173305644 \\ \mathrm{H} & -2.0269998284 & -0.4327993924 & -0.4785672253 \\ \mathrm{H} & 1.2242018954 & 0.8257102533 & -1.8100648747 \\ \mathrm{H} & 1.7216281699 & 1.7199951656 & -0.3586942230 \\ \mathrm{C} & 1.9708048906 & -0.4755622542 & -0.1769538947 \\ \mathrm{H} & 2.3590566872 & -1.0704740627 & -1.0201880742 \\ \mathrm{H} & 2.8406346886 & -0.1788711416 & 0.4396532888 \\ \mathrm{C} & 0.9708983446 & -1.3366985364 & 0.6462996918 \\ \mathrm{H} & 1.3671379271 & -0.1595643947 & 2.4708508764 \\ \mathrm{H} & -0.1417591654 & -1.0996640539 & 2.5128800635 \\ \mathrm{Cl} & -1.0869840010 & 2.3231796248 & -0.8154176954 \\ \mathrm{H} & 1.4574071900 & -2.2650305232 & 0.9899671876 \\ \mathrm{O} & -0.6274117345 & -2.8992620016 & -0.5094668280\end{array}$

\section{Chloro-byciclo[7.3.1.0]tridecan-13-one (4b)}

\section{Neutral}

UBPW91/6-31+G*, -1042.20166708409
xyz coordinates:

$\begin{array}{lr}\mathrm{C} & -2.3329570000 \\ \mathrm{C} & -2.4233160000 \\ \mathrm{C} & -1.0572460000 \\ \mathrm{C} & -0.2515920000 \\ \mathrm{C} & -0.2300070000 \\ \mathrm{C} & -1.6114600000 \\ \mathrm{C} & 1.2064190000 \\ \mathrm{C} & 1.8962880000 \\ \mathrm{C} & 1.2588690000 \\ \mathrm{C} & -0.2190480000 \\ \mathrm{C} & 1.4234710000 \\ \mathrm{C} & 0.9955950000 \\ \mathrm{C} & 1.4645580000 \\ \mathrm{O} & 2.9018680000 \\ \mathrm{C} & -1.1383870000 \\ \mathrm{H} & -3.3487090000 \\ \mathrm{H} & -1.7964110000 \\ \mathrm{H} & -2.2237020000 \\ \mathrm{H} & -1.4846610000 \\ \mathrm{H} & 0.2747020000 \\ \mathrm{H} & 0.4119200000 \\ \mathrm{H} & 1.7256630000 \\ \mathrm{H} & -0.2062800000 \\ \mathrm{H} & -0.7301200000 \\ \mathrm{H} & -3.0908990000 \\ \mathrm{H} & -2.8874960000\end{array}$

$$
\begin{array}{r}
-0.4310150000 \\
-0.6446330000 \\
-0.5704700000 \\
0.7001850000 \\
0.9434600000 \\
0.8863140000 \\
0.7150580000 \\
-0.5596400000 \\
-1.8130230000 \\
-1.8585770000 \\
0.7661570000 \\
-0.5013520000 \\
-1.7996560000 \\
-0.5485500000 \\
2.1847140000 \\
-0.4295260000 \\
-1.2726950000 \\
1.7426910000 \\
0.9976050000 \\
1.9026750000 \\
0.1591240000 \\
1.5800590000 \\
-2.1048520000 \\
-2.6984870000 \\
0.1231600000 \\
-1.6212380000
\end{array}
$$

-2.1176980000
-0.5958820000
0.1357980000
-0.2498660000
-1.7753770000
-2.4475210000
0.3044420000
-0.1700170000
0.3817660000
-0.1003840000
1.8522190000
2.6183970000
1.9299810000
-0.8899770000
0.5108810000
-2.5508570000
-2.5942460000
-2.1124930000
-3.5386210000
-1.9833030000
-2.2218420000
-0.1416170000
-1.1765140000
0.4028250000
-0.1658270000
-0.3694120000

\section{S13}




$\begin{array}{rrrr}\mathrm{H} & 1.7877940000 & -2.6882910000 & -0.0299970000 \\ \mathrm{H} & 2.5454380000 & -1.9399430000 & 2.1147360000 \\ \mathrm{H} & 0.9557600000 & -2.6792280000 & 2.3627450000 \\ \mathrm{H} & 2.5060920000 & 0.9324650000 & 2.0012040000 \\ \mathrm{H} & 0.9159060000 & 1.6583780000 & 2.2538570000 \\ \mathrm{H} & -0.0964660000 & -0.5138960000 & 2.7598560000 \\ \mathrm{H} & 1.4249400000 & -0.4592140000 & 3.6354280000 \\ \mathrm{H} & -1.2763820000 & -0.5096590000 & 1.2117280000\end{array}$

\section{Radical}

UBPW91/6-31+G*, $\quad-581.94422773962$

xyz coordinates:

\begin{tabular}{|c|c|c|c|}
\hline C & 0.0032505181 & -0.0079241862 & -0.0152277508 \\
\hline $\mathrm{C}$ & 0.0044819469 & 0.0204914041 & 1.5359167405 \\
\hline $\mathrm{C}$ & 1.3890310562 & 0.0312692315 & 2.1489360449 \\
\hline C & 2.6106720760 & -0.4272242095 & 1.3945831862 \\
\hline C & 2.4923804790 & -0.3385867844 & -0.138815891 \\
\hline C & 1.1302643098 & -0.8639434666 & -0.61522659 \\
\hline C & 1.4874333130 & 0.0158606281 & 3.66250875 \\
\hline $\mathrm{C}$ & 0.9098519506 & -1.3784832754 & 3.88546755 \\
\hline $\mathrm{C}$ & -0.5892994253 & -1.4458773770 & 3.63629957 \\
\hline $\mathrm{C}$ & -0.8283458358 & -1.1713756472 & 2.12308211 \\
\hline C & 0.7371618888 & 1.0931681494 & 4.50061781 \\
\hline C & -0.8033206921 & 0.9885813604 & 4.49988135 \\
\hline C & -1.3001146038 & -0.4663753458 & 4.61241324 \\
\hline O & 1.6157005516 & -2.3733043298 & 4.13592410 \\
\hline $\mathrm{H}$ & 1.0714874956 & -0.8380447861 & -1.7177582 \\
\hline $\mathrm{H}$ & 1.0145048434 & -1.9249572661 & -0.32035 \\
\hline $\mathrm{H}$ & 2.6073879737 & 0.7130206792 & -0 . \\
\hline $\mathrm{H}$ & 3.3198777487 & -0.9042674559 & -0.601 \\
\hline $\mathrm{H}$ & 3.4989753848 & 0.1324778190 & 1.7 \\
\hline $\mathrm{H}$ & 2.8252636310 & -1.4872376397 & 1.66 \\
\hline $\mathrm{H}$ & 2.5497431026 & 0.0087046825 & 3.95 \\
\hline $\mathrm{H}$ & -0.5515174428 & -2.0895187555 & 1.5 \\
\hline $\mathrm{H}$ & -1.9054106357 & -0.9992936560 & 1.9 \\
\hline $\mathrm{H}$ & 0.1155842385 & 1.0265804116 & -0.3 \\
\hline $\mathrm{H}$ & -0.9849673370 & -0.3583950512 & -0.3 \\
\hline $\mathrm{H}$ & -0.9267267441 & -2.4719661855 & 3. \\
\hline $\mathrm{H}$ & -1.1267691299 & -0.8361912335 & 5.64078927 \\
\hline $\mathrm{H}$ & -2.3907167080 & -0.5156357760 & 4.44334794 \\
\hline $\mathrm{H}$ & 1.0996145690 & 0.9747003395 & 5.53867164 \\
\hline $\mathrm{H}$ & 1.0585501808 & 2.0966954841 & 4.16954479 \\
\hline $\mathrm{H}$ & -1.2243544149 & 1.4592309653 & 3.5968542 \\
\hline $\mathrm{H}$ & -1.1979089378 & 1.5734949 & 5.3503800 \\
\hline $\mathrm{H}$ & -0.5135896 & 0.9491878801 & 1.836 \\
\hline
\end{tabular}

\section{Chloro-byciclo[2.2.1]heptane (1a)}

\section{neutral}

UBPW91/6-31+G*, -733.53313839267

xyz coordinates:
C $\quad-0.2447010064$
$-0.3189284559$
$-0.2820754830$
$-0.3388405561$
$-1.4083434776$
0.1365927732
C $\quad 1.2106722734$
$-0.2828179420$
0.5193353634 


\begin{tabular}{|c|c|c|c|}
\hline $\mathrm{C}$ & 1.4373414496 & 1.0375272054 & -0.2574085393 \\
\hline C & 0.3748735735 & 1.9694835131 & 0.3892387994 \\
\hline $\mathrm{C}$ & -0.8276152074 & 1.0046733083 & 0.6712902745 \\
\hline $\mathrm{H}$ & 1.7874981893 & -1.1368378621 & 0.1271005549 \\
\hline $\mathrm{H}$ & 1.3729914321 & -0.1953906244 & 1.6064933087 \\
\hline $\mathrm{H}$ & 1.7566599775 & 0.1312013489 & -2.2368180338 \\
\hline $\mathrm{H}$ & 0.1007807501 & 2.8063128146 & -0.2739233042 \\
\hline $\mathrm{H}$ & 0.7564720072 & 2.4018531700 & 1.3292390504 \\
\hline $\mathrm{H}$ & 0.6620553694 & 1.5326875673 & -2.2747520950 \\
\hline $\mathrm{H}$ & -1.2002167079 & 0.0289291299 & -1.8333059719 \\
\hline $\mathrm{H}$ & -0.0494793488 & -1.3292441101 & -1.8029598647 \\
\hline $\mathrm{Cl}$ & -1.2064959504 & -1.7781394697 & 0.7936066333 \\
\hline
\end{tabular}

\section{radical}

UBPW91/6-31+G*, -273.2538990815

xyz coordinates:

$\begin{array}{lrrr}\mathrm{C} & -0.4915713607 & -0.8626071567 & -1.0119443842 \\ \mathrm{C} & -0.5395171662 & -0.8844100963 & 0.5152585911 \\ \mathrm{C} & 0.9204452470 & -0.8975064255 & 0.9462360928 \\ \mathrm{C} & 1.5389749392 & 0.0947719097 & -0.1246701591 \\ \mathrm{C} & 0.3089783354 & 0.4795313306 & -1.0008498701 \\ \mathrm{C} & -0.6422478576 & 1.3964159127 & -0.1743613563 \\ \mathrm{C} & -1.2581130171 & 0.4023538007 & 0.8963888492 \\ \mathrm{H} & -1.4771888284 & -0.7745371989 & -1.4994420680 \\ \mathrm{H} & 0.5784734544 & 0.8935191028 & -1.9854917531 \\ \mathrm{H} & 0.0698506837 & -1.6978228752 & -1.4637160192 \\ \mathrm{H} & -1.4306897661 & 1.8240112370 & -0.8168683764 \\ \mathrm{H} & 2.0078790956 & 0.9769308175 & 0.3428875616 \\ \mathrm{H} & 1.3737793339 & -1.9002084199 & 0.8597900380 \\ \mathrm{H} & 1.0683218673 & -0.5532244634 & 1.9829853610 \\ \mathrm{H} & 2.3108310745 & -0.4086297650 & -0.7313970734 \\ \mathrm{H} & -0.1044601442 & 2.2375012715 & 0.2948323300 \\ \mathrm{H} & -1.0601351211 & 0.7166420267 & 1.9342884957 \\ \mathrm{H} & -2.3523677416 & 0.3231882147 & 0.7748532595\end{array}$

\section{Chloro-adamantane (2a)}

\section{neutral}

UBPW91/6-31+G*, $\quad-850.27680577532$

xyz coordinates:

$\begin{array}{lr}\text { C } & -0.4660139220 \\ \text { C } & -0.4530686860 \\ \text { C } & 1.0071762764 \\ \text { C } & 1.7421829588 \\ \text { C } & 1.7295259719 \\ \text { C } & 0.2692441459 \\ \text { C } & -1.1752417172 \\ \text { C } & -0.4250212733 \\ \text { C } & 1.0203321265 \\ \text { C } & -0.4529879370\end{array}$

1.7717648895

1.7467852451

1.7401573397

0.4943470929

0.5193134029

0.5262029404

0.4650264597

$-0.7537013989$

$-0.7872829036$

$-0.7556367522$
-1.1940662261
0.3518009573
0.8600306171
0.3129183029
-1.2329264911
-1.7407512965
0.8501103953
0.2928424756
0.8112930803
-1.2425071239

\section{S15}




$\begin{array}{lr}\mathrm{Cl} & -1.2987390371 \\ \mathrm{H} & -1.5080248860 \\ \mathrm{H} & 0.2536261294 \\ \mathrm{H} & 2.2667745436 \\ \mathrm{H} & 2.2598072974 \\ \mathrm{H} & 2.7830823089 \\ \mathrm{H} & 1.0244249962 \\ \mathrm{H} & 1.5246078259 \\ \mathrm{H} & -0.9868614540 \\ \mathrm{H} & -1.4967557649 \\ \mathrm{H} & 0.0528441226 \\ \mathrm{H} & -2.2244616814 \\ \mathrm{H} & -1.1843111297 \\ \mathrm{H} & 1.5376981316 \\ \mathrm{H} & 1.0274607540 \\ \mathrm{H} & 0.0274252454\end{array}$
$-2.3067140772$
1. 7919030808
0.5318922478
$-0.3619936108$
1. 4179111645
0.4760689419
1. 7380426200
2. 6595757045
2.6283441510
$-0.7733363758$
$-1.6579451993$
0.4563639188
0.4333849812
$-1.6890505147$
$-0.8276904295$
2. 6915293259

0.8968503237

$-1.5656976437$

$-2.8449487604$

$-1.6318225825$

$-1.5996041741$

0.6819704376

1. 9664888892

0.5282506836

0.7486404307

$-1.6038483291$

$-1.6304745115$

0.5046048064

1. 9543854020

0.4378109490

1.9153187992

$-1.5597618686$

\section{radical}

UBPW91/6-31+G*, -390.00487503839

xyz coordinates:

$\begin{array}{lr}\mathrm{C} & -1.0747088562 \\ \mathrm{C} & 0.4940331156 \\ \mathrm{C} & 0.9991315878 \\ \mathrm{C} & 0.5008658245 \\ \mathrm{C} & -1.0681676312 \\ \mathrm{C} & -1.4743991846 \\ \mathrm{C} & 0.9991315878 \\ \mathrm{C} & 0.5008658245 \\ \mathrm{C} & 1.0063915860 \\ \mathrm{C} & -1.0681676312 \\ \mathrm{H} & 0.6465784481 \\ \mathrm{H} & 0.8572692117 \\ \mathrm{H} & 0.6465784481 \\ \mathrm{H} & 2.1049454311 \\ \mathrm{H} & 0.8685244831 \\ \mathrm{H} & 0.6600001058 \\ \mathrm{H} & 2.1124453773 \\ \mathrm{H} & 0.8685244831 \\ \mathrm{H} & -1.4505288882 \\ \mathrm{H} & -1.4505288882 \\ \mathrm{H} & -1.4436204443 \\ \mathrm{H} & -1.4392331687 \\ \mathrm{H} & -1.4436204443 \\ \mathrm{H} & -1.4392331687 \\ \mathrm{H} & 2.1049454311\end{array}$

$$
\begin{array}{r}
1.4521154010 \\
1.4682834190 \\
0.7335978617 \\
-0.7318822712 \\
-0.7311175927 \\
-0.0047001397 \\
0.7335978617 \\
-0.7318822712 \\
-1.4575317019 \\
-0.7311175927 \\
1.2573479208 \\
2.5130079149 \\
1.2573479208 \\
0.7525392645 \\
-1.2525227346 \\
-2.5087267965 \\
-1.4843086263 \\
-1.2525227346 \\
1.9859956479 \\
1.9859956479 \\
-0.2260600985 \\
-1.7734937657 \\
-0.2260600985 \\
-1.7734937657 \\
0.7525392645
\end{array}
$$

0.0000000000

0.0000000000

1.2651900166

1. 2703620237

1.2603007881

0.0000000000

$-1.2651900166$

$-1.2703620237$

0.0000000000

$-1.2603007881$

2.1741835234

0.0000000000

$-2.1741835234$

$-1.2923061818$

$-2.1742837194$

0.0000000000

0.0000000000

2.1742837194

0.8934116074

$-0.8934116074$

2.1705061669

1.2769965148

$-2.1705061669$

$-1.2769965148$

1.2923061818

\section{Cloro-byciclo[2.2.2]octane}

\section{(3a)}

\section{neutral}

UBPW91/6-31+G*, $\quad-772.84764743457$

xyz coordinates:
C $\quad 0.0000000000$
0.0000000000
0.0000000000
C $\quad 0.0000000000$
0.0000000000
1.5443726265 


\begin{tabular}{|c|c|c|c|}
\hline $\mathrm{C}$ & 1.4726983808 & 0.0000000000 & 2.0754769383 \\
\hline C & 2.4208220561 & 0.0308470382 & 0.8653120820 \\
\hline C & 2.1862957975 & 1.3006204605 & 0.0317443588 \\
\hline C & 0.7182732028 & 1.2679306721 & -0.5101258803 \\
\hline C & 0.7506626047 & -1.2496211928 & -0.5101752570 \\
\hline C & 2.2302784063 & -1.2256698502 & 0.0004994279 \\
\hline $\mathrm{Cl}$ & 4.1770759033 & 0.0532605204 & 1.4930804044 \\
\hline $\mathrm{H}$ & 1.6654179403 & 0.8759736137 & 2.7175394204 \\
\hline $\mathrm{H}$ & 1.6793008322 & -0.8980830973 & 2.6817170010 \\
\hline $\mathrm{H}$ & 2.9157434145 & 1.3409805971 & -0.7946066353 \\
\hline $\mathrm{H}$ & 2.3580281480 & 2.1901850062 & 0.6607886981 \\
\hline $\mathrm{H}$ & 2.9430658756 & -1.2050808313 & -0.8410914322 \\
\hline $\mathrm{H}$ & 2.4609007598 & -2.1229658491 & 0.5990199454 \\
\hline $\mathrm{H}$ & 0.7276327212 & -1.2756005679 & -1.6132442320 \\
\hline $\mathrm{H}$ & 0.2389634525 & -2.1616632483 & -0.1577024579 \\
\hline $\mathrm{H}$ & -0.5409661571 & -0.8864586660 & 1.9177294834 \\
\hline $\mathrm{H}$ & -0.5415510158 & 0.8862801905 & 1.9177291436 \\
\hline $\mathrm{H}$ & -1.0388062111 & -0.0130985321 & -0.3714762605 \\
\hline $\mathrm{H}$ & 0.7168327428 & 1.2786068940 & -1.6133877518 \\
\hline $\mathrm{H}$ & 0.1707622537 & 2.1675508652 & -0.1803743127 \\
\hline
\end{tabular}

\section{radical}

UBPW91/6-31+G*, $\quad-312.57381233204$

xyz coordinates:

$\begin{array}{rrrr}\mathrm{C} & 0.000000 & 0.000000 & 0.000000 \\ \mathrm{C} & 0.000000 & 0.000000 & 1.511292 \\ \mathrm{C} & 1.393511 & 0.000000 & 2.096661 \\ \mathrm{C} & 2.053460 & -1.373857 & 1.665896 \\ \mathrm{C} & 1.037130 & -2.182698 & 0.822321 \\ \mathrm{C} & 0.653258 & -1.373746 & -0.441509 \\ \mathrm{C} & -0.880325 & -1.080106 & 2.096425 \\ \mathrm{C} & -0.232325 & -2.459785 & 1.665357 \\ \mathrm{H} & 1.370323 & 0.085001 & 3.197580 \\ \mathrm{H} & 1.992011 & 0.847420 & 1.717707 \\ \mathrm{H} & -1.915206 & -1.008640 & 1.717143 \\ \mathrm{H} & -0.931757 & -1.008579 & 3.197342 \\ \mathrm{H} & -1.024037 & 0.084531 & -0.405046 \\ \mathrm{H} & 0.580781 & 0.847608 & -0.405006 \\ \mathrm{H} & -0.051120 & -1.957036 & -1.060660 \\ \mathrm{H} & 1.550647 & -1.195652 & -1.060125 \\ \mathrm{H} & 2.971212 & -1.196165 & 1.078007 \\ \mathrm{H} & 2.351359 & -1.956984 & 2.555192 \\ \mathrm{H} & 1.492564 & -3.140698 & 0.519896 \\ \mathrm{H} & -0.950049 & -3.058299 & 1.076953 \\ \mathrm{H} & 0.030713 & -3.059467 & 2.554653\end{array}$

\section{Cloro-byciclo[7.3.1.0]tridecane (4a)}

\section{neutral}

UBPW91/6-31+G*, -968.17409182912

xyz coordinates: 

0.0108628824
0.0502762070
0.1035981491
$-1.2113441040$
$-1.2510082836$
$-1.0951377701$
$-1.1213281334$
$-2.5110236240$
$-2.4049096239$
$-2.4148000162$
$-3.5250128923$
$-3.4932880658$
$-3.5857521335$
$-4.0015181572$
$-4.3260085926$
$-2.5619587948$
$-4.5114561185$
$-3.3978824573$
$-2.4605706085$
$-1.4403773273$
$-1.1815994226$
$-0.8635498562$
$-1.2189144938$
$-4.5841357877$
$-3.5099311060$
0.9787087606
0.9950083703
$-0.0851540661$
$-0.4119694222$
$-2.1711167935$
$-2.0578466780$
$-0.8659111711$
$-2.8333101289$
0.2691153877
0.9524094417
1. 4695312642
1. 9887662619
1.5966853661
0.0459756274
$-0.6867910155$
2.1625393054
2. 0321895115
2. 3151031826
3. 8250579023
4.6280546491
4.4036275910
2. 9037205291
1. 6301336237
4.9437524065
4. 8323127988
4.3310927011
5.6991476309
3. 9567952409
4. 2380465656
1. 9754526408
3. 2323983422
1. 7608438995
2.5357989060
2.7423090572
1.7679646499
$-0.4275851279$
$-0.4637764614$
$-0.2436893242$
$-0.2903740015$
$-0.6921210404$
$-1.7506384774$
0.9872579122
3. 0796135202
1.5350061712

\section{radical}

UBPW91/6-31+G*, $\quad-507.91153809639$

xyz coordinates:

\begin{tabular}{lrrr}
$\mathrm{C}$ & 0.0236896397 & -0.0542922438 & -0.1811522768 \\
$\mathrm{C}$ & -0.0875938634 & 0.0637231219 & 1.3608968029 \\
$\mathrm{C}$ & 1.3276324255 & 0.1957331233 & 1.9571168169 \\
$\mathrm{C}$ & 2.1037878155 & -1.1266804413 & 1.6822199804 \\
$\mathrm{C}$ & 2.2715764460 & -1.2819211588 & 0.1419908730 \\
$\mathrm{C}$ & 0.9628808200 & -1.1794035672 & -0.6747450477 \\
$\mathrm{C}$ & -0.8406304649 & -1.0936238419 & 2.0637900812 \\
$\mathrm{C}$ & -0.0755027013 & -2.4607871078 & 2.0743685496 \\
$\mathrm{C}$ & 1.3864987337 & -2.2437075764 & 2.4181883259 \\
$\mathrm{C}$ & 1.9184704495 & -2.5799008314 & 3.7916418642 \\
$\mathrm{C}$ & 1.1722429033 & -3.7151505747 & 4.5161813540 \\
$\mathrm{C}$ & -0.3464598428 & -3.5013479210 & 4.4303267384 \\
$\mathrm{C}$ & -0.7899549885 & -3.5166513687 & 2.9589322404 \\
$\mathrm{H}$ & -0.8830121399 & -4.2862647758 & 4.9935328562 \\
$\mathrm{H}$ & -0.6120248939 & -2.5371660003 & 4.9065863530 \\
$\mathrm{H}$ & 1.4269562257 & -4.6883198239 & 4.0531008221 \\
$\mathrm{H}$ & 1.5054869684 & -3.7680297946 & 5.5680171434 \\
$\mathrm{H}$ & 3.0019573416 & -2.8065650040 & 3.7302394040 \\
$\mathrm{H}$ & 1.8651000453 & -1.6708438577 & 4.4406043489 \\
$\mathrm{H}$ & 3.1220469972 & -1.0345491675 & 2.1063451535 \\
\hline \hline
\end{tabular}




$\begin{array}{lr}\mathrm{H} & -1.0319628759 \\ \mathrm{H} & -1.8312002433 \\ \mathrm{H} & -0.5792239034 \\ \mathrm{H} & -1.8836372878 \\ \mathrm{H} & -0.6543920200 \\ \mathrm{H} & 0.4047430249 \\ \mathrm{H} & -0.9806542400 \\ \mathrm{H} & 2.9573511519 \\ \mathrm{H} & 2.7828405666 \\ \mathrm{H} & 0.4322474257 \\ \mathrm{H} & 1.2130294069 \\ \mathrm{H} & -0.1362218546 \\ \mathrm{H} & 1.2754200817 \\ \mathrm{H} & 1.8617175039\end{array}$

$-0.7887128081$ $-1.2447598188$ $-4.5234613340$ $-3.3737743437$ 0.9908037474 0.9162181333 $-0.1833505144$ $-0.4752916577$ $-2.2345944222$ $-2.1454593867$ $-1.0046968581$ $-2.8433791566$ 0.3883830972 1.0514383586
3. 1111576642 1.5924397036

2. 5495002862

2.8779597940

1.5722781968

$-0.5550384097$

$-0.6261384874$

$-0.1838108282$

$-0.0875118379$

$-0.6578643614$

$-1.7376955664$

1.0393204240

3.0446592439

1.4997664060 


\section{4-Diphenylphanyl-bicyclo[2.2.1]heptan-2-one (7)}

UBPW91/6-31+G*, -1151.95446826965

xyz coordinates:

$\begin{array}{rrrr}\text { C } & -0.058077 & -0.085032 & 0.037170 \\ \mathrm{C} & -0.047527 & 0.005168 & 1.596132 \\ \mathrm{C} & 1.433061 & 0.064071 & 2.055686 \\ \mathrm{C} & 1.878468 & 1.442008 & 1.551542 \\ \mathrm{C} & 0.656015 & 2.094187 & 0.933605 \\ \mathrm{C} & 0.436726 & 1.328510 & -0.415115 \\ \mathrm{C} & -0.472362 & 1.494170 & 1.807981 \\ \mathrm{P} & -1.255508 & -1.217980 & 2.381164 \\ \mathrm{C} & -0.740170 & -2.890797 & 1.755741 \\ \mathrm{C} & -1.786189 & -3.681612 & 1.224428 \\ \mathrm{C} & -1.532225 & -4.955535 & 0.687563 \\ \mathrm{C} & -0.221091 & -5.459475 & 0.668754 \\ \mathrm{C} & 0.829698 & -4.684981 & 1.192016 \\ \mathrm{C} & 0.573943 & -3.413478 & 1.732358 \\ \mathrm{O} & 3.021503 & 1.900827 & 1.618548 \\ \mathrm{C} & -0.815368 & -1.193443 & 4.190133 \\ \mathrm{C} & -0.101571 & -2.204507 & 4.870189 \\ \mathrm{C} & 0.094832 & -2.134257 & 6.261229 \\ \mathrm{C} & -0.410564 & -1.049613 & 6.997159 \\ \mathrm{C} & -1.128938 & -0.038831 & 6.334409 \\ \mathrm{C} & -1.340779 & -0.118333 & 4.948942 \\ \mathrm{H} & 2.088937 & -0.725951 & 1.656053 \\ \mathrm{H} & -0.417559 & 1.820622 & 2.859464 \\ \mathrm{H} & 0.721432 & 3.186877 & 0.839982 \\ \mathrm{H} & -1.477306 & 1.715329 & 1.411922 \\ \mathrm{H} & 1.348671 & 1.289609 & -1.032419 \\ \mathrm{H} & -1.075370 & -0.282816 & -0.340862 \\ \mathrm{H} & 0.591931 & -0.898475 & -0.320979 \\ \mathrm{H} & -0.335477 & 1.858748 & -0.996788 \\ \mathrm{H} & -2.810886 & -3.292777 & 1.224779 \\ \mathrm{H} & -2.358289 & -5.548837 & 0.280137 \\ \mathrm{H} & -0.016613 & -6.449243 & 0.246708 \\ \mathrm{H} & 1.854390 & -5.072312 & 1.181111 \\ \mathrm{H} & 1.409249 & -2.836072 & 2.137475 \\ \mathrm{H} & -1.943390 & 0.654690 & 4.458711 \\ \mathrm{H} & -1.543101 & 0.804350 & 6.897988 \\ \mathrm{H} & -0.258006 & -0.998527 & 8.080516 \\ \mathrm{H} & 0.644019 & -2.933761 & 6.770611 \\ \mathrm{H} & 0.293844 & -3.062906 & 4.320252 \\ \mathrm{H} & 1.517558 & 0.048714 & 3.158435\end{array}$

\section{Radical Anion of 4-Diphenylphanyl-bicyclo[2.2.1] heptan-2-one (7•-)}

UBPW91/6-31+G*, -1152.01889249992

xyz coordinates:

$\begin{array}{lrrr}\text { C } & -0.0199999122 & -0.0399493362 & 0.0216074135 \\ \text { C } & -0.0350514768 & -0.0017180392 & 1.5821049864 \\ \text { C } & 1.4267066831 & -0.0033216324 & 2.0851961323 \\ \text { C } & 1.9119667049 & 1.3589503020 & 1.6054345108 \\ \text { C } & 0.7112423409 & 2.0724334820 & 0.9924701998\end{array}$




\begin{tabular}{lrrr}
$\mathrm{C}$ & 0.4520480135 & 1.3968592101 & -0.3895183322 \\
$\mathrm{C}$ & -0.4436365484 & 1.4812091042 & 1.8315890356 \\
$\mathrm{P}$ & -1.2535283632 & -1.2622857339 & 2.3250589211 \\
$\mathrm{C}$ & -0.7643920303 & -2.8738467673 & 1.5898714575 \\
$\mathrm{C}$ & -1.7767681630 & -3.5755127864 & 0.8862064018 \\
$\mathrm{C}$ & -1.5089629443 & -4.7777876665 & 0.2163634396 \\
$\mathrm{C}$ & -0.1999141653 & -5.3174429419 & 0.2313204040 \\
$\mathrm{C}$ & 0.8177229127 & -4.6334163108 & 0.9186927395 \\
$\mathrm{C}$ & 0.5504895899 & -3.4268082452 & 1.5898443954 \\
$\mathrm{O}$ & 3.1426980481 & 1.6813847668 & 1.4190445238 \\
$\mathrm{C}$ & -0.7726905520 & -1.3125891654 & 4.1188213862 \\
$\mathrm{C}$ & -0.2099170203 & -2.4365170548 & 4.7746668824 \\
$\mathrm{C}$ & -0.0390347288 & -2.4586451245 & 6.1674690350 \\
$\mathrm{C}$ & -0.4231432452 & -1.3520912683 & 6.9561177125 \\
$\mathrm{C}$ & -0.9985444664 & -0.2337002447 & 6.3244051589 \\
$\mathrm{C}$ & -1.1831676542 & -0.2168458687 & 4.9336766465 \\
$\mathrm{H}$ & 2.0330577117 & -0.8226681512 & 1.6491939869 \\
$\mathrm{H}$ & -0.3885896419 & 1.7733816974 & 2.8925386050 \\
$\mathrm{H}$ & 0.8003892449 & 3.1710570819 & 0.9512758038 \\
$\mathrm{H}$ & -1.4439517095 & 1.7236492324 & 1.4329171697 \\
$\mathrm{H}$ & 1.3580829675 & 1.3828452769 & -1.0178034872 \\
$\mathrm{H}$ & -1.0265128112 & -0.2547406801 & -0.3777181495 \\
$\mathrm{H}$ & 0.6518500719 & -0.8331304466 & -0.3438352090 \\
$\mathrm{H}$ & -0.3322340514 & 1.9363722678 & -0.9546389407 \\
$\mathrm{H}$ & -2.7928348351 & -3.1634656155 & 0.8623979230 \\
$\mathrm{H}$ & -2.3143819600 & -5.2935651153 & -0.3188144958 \\
$\mathrm{H}$ & 0.0180029491 & -6.2565806686 & -0.2882249409 \\
$\mathrm{H}$ & 1.8349122091 & -5.0428071982 & 0.9339402670 \\
$\mathrm{H}$ & 1.3622515734 & -2.9191511214 & 2.1171577661 \\
$\mathrm{H}$ & -1.6688367038 & 0.6525860152 & 4.4769823192 \\
$\mathrm{H}$ & -1.3183443847 & 0.6295291308 & 6.9199873454 \\
$\mathrm{H}$ & -0.2896028279 & -1.3694119329 & 8.0430614500 \\
$\mathrm{H}$ & 0.3979213081 & -3.3443371262 & 6.6429055000 \\
$\mathrm{H}$ & 0.0879943573 & -3.3107331449 & 4.1882674693 \\
$\mathrm{H}$ & 1.4613918092 & -0.1335808862 & 3.1887015303 \\
& & & \\
\hline
\end{tabular}

\section{Neutral molecule of Bicyclo[2.2.1]hept-1-yl-diphenyl-phosphane ( 8 )}

\begin{tabular}{|c|c|c|c|}
\hline $\mathrm{C}$ & 0.0589055092 & -0.0126218990 & 0.0040163724 \\
\hline C & -0.0689183307 & 0.0891633920 & 1.5553356185 \\
\hline $\mathrm{C}$ & 1.3672903899 & 0.1910090693 & 2.1522649838 \\
\hline $\mathrm{C}$ & 1.8351087196 & 1.6315315756 & 1.7443682121 \\
\hline $\mathrm{C}$ & 0.6363892321 & 2.1748980731 & 0.9298537966 \\
\hline $\mathrm{C}$ & 0.5587037450 & 1.4128192290 & -0.4163805226 \\
\hline $\mathrm{C}$ & -0.5553204355 & 1.5651811008 & 1.7021369993 \\
\hline $\mathrm{P}$ & -1.3553039776 & -1.1217735692 & 2.2320814415 \\
\hline $\mathrm{C}$ & -0.7875873968 & -2.8271177159 & 1.7531361666 \\
\hline $\mathrm{C}$ & -1.8213367839 & -3.7732363740 & 1.5659741434 \\
\hline $\mathrm{C}$ & -1.5366079039 & -5.0984845590 & 1.2040501150 \\
\hline $\mathrm{C}$ & -0.2076078516 & -5.5012561514 & 1.0014238091 \\
\hline $\mathrm{C}$ & 0.8305805635 & -4.5722050262 & 1.1712079562 \\
\hline $\mathrm{C}$ & 0.5441731499 & -3.2503994751 & 1.5486392624 \\
\hline $\mathrm{C}$ & -1.0411980819 & -1.0863747941 & 4.0681584353 \\
\hline $\mathrm{C}$ & -0.2250440219 & -2.0002828405 & 4.7680279966 \\
\hline $\mathrm{C}$ & -0.0790707033 & -1.9101579861 & 6.1615144530 \\
\hline $\mathrm{C}$ & -0.7404822010 & -0.9029956444 & 6.8812680019 \\
\hline C & -1.5625222769 & 0.0077757145 & 6.1988746341 \\
\hline
\end{tabular}




$\begin{array}{rrrr}\mathrm{C} & -1.7201641424 & -0.0917434969 & 4.8084357091 \\ \mathrm{H} & -0.6075310485 & 1.8998894040 & 2.7521687972 \\ \mathrm{H} & 0.6296125797 & 3.2716221544 & 0.8240670204 \\ \mathrm{H} & -1.5320881915 & 1.7393019159 & 1.2193150949 \\ \mathrm{H} & 2.0143207640 & 2.2546082476 & 2.6375464578 \\ \mathrm{H} & 1.5334741530 & 1.3832387252 & -0.9325532695 \\ \mathrm{H} & -0.9194626737 & -0.2394425906 & -0.4546531082 \\ \mathrm{H} & 0.7510233825 & -0.8158560482 & -0.2956364865 \\ \mathrm{H} & -0.1593537526 & 1.8961815732 & -1.1007823693 \\ \mathrm{H} & 2.7690189190 & 1.6239678060 & 1.1566930310 \\ \mathrm{H} & -2.8619459697 & -3.4604789458 & 1.7020772290 \\ \mathrm{H} & -2.3544364761 & -5.8131944976 & 1.0693686757 \\ \mathrm{H} & 0.0181727152 & -6.5314310642 & 0.7095234795 \\ \mathrm{H} & 1.8703290984 & -4.8770557628 & 1.0143355293 \\ \mathrm{H} & 1.3693604390 & -2.5490071611 & 1.6876236407 \\ \mathrm{H} & -2.3930940710 & 0.6004356466 & 4.2910900553 \\ \mathrm{H} & -2.0958286348 & 0.7879328546 & 6.7512623581 \\ \mathrm{H} & -0.6259866946 & -0.8355601660 & 7.9674481444 \\ \mathrm{H} & 0.5553694870 & -2.6319104850 & 6.6861566087 \\ \mathrm{H} & 0.2933725042 & -2.7953892971 & 4.2249205588 \\ \mathrm{H} & 1.3494852661 & 0.0718302756 & 3.2474250803 \\ \mathrm{H} & 2.0381034046 & -0.5830922647 & 1.7459876172\end{array}$

\section{Radical Anion of Bicyclo[2.2.1]hept-1-yl-diphenyl-phosphane ( 8•- )}

\begin{tabular}{|c|c|c|c|}
\hline C & 0.0776262746 & -0.0103714740 & -0.0310294476 \\
\hline $\mathrm{C}$ & 0.0768220030 & 0.0342260141 & 1.5257510106 \\
\hline $\mathrm{C}$ & 1.5603922754 & 0.0359586442 & 1.9957305357 \\
\hline $\mathrm{C}$ & 2.0859823960 & 1.4544842293 & 1.5747971875 \\
\hline $\mathrm{C}$ & 0.8467039889 & 2.1056443007 & 0.9119236108 \\
\hline $\mathrm{C}$ & 0.5893442603 & 1.4118985400 & -0.4487223642 \\
\hline $\mathrm{C}$ & -0.3018988141 & 1.5269179200 & 1.7687407954 \\
\hline $\mathrm{P}$ & -1.2158926346 & -1.1494128375 & 2.2729865701 \\
\hline $\mathrm{C}$ & -0.8533846851 & -2.8138322710 & 1.5914632655 \\
\hline $\mathrm{C}$ & -1.9690073448 & -3.5703049484 & 1.1205548274 \\
\hline $\mathrm{C}$ & -1.8496742437 & -4.8927693257 & 0.6823220985 \\
\hline $\mathrm{C}$ & -0.5799983255 & -5.5421029915 & 0.7025713987 \\
\hline $\mathrm{C}$ & 0.5383090818 & -4.8142975695 & 1.1585265459 \\
\hline $\mathrm{C}$ & 0.4200071921 & -3.4826991373 & 1.5888507127 \\
\hline $\mathrm{C}$ & -0.8548853757 & -1.2095540487 & 4.0732326976 \\
\hline $\mathrm{C}$ & 0.2709302574 & -1.8614239628 & 4.6921340670 \\
\hline $\mathrm{C}$ & 0.3425889215 & -2.0271296011 & 6.0811423618 \\
\hline C & -0.6725952512 & -1.5365818886 & 6.9360644747 \\
\hline C & -1.7868449900 & -0.8722450008 & 6.3467561337 \\
\hline $\mathrm{C}$ & -1.8706114217 & -0.7256888322 & 4.9601209885 \\
\hline $\mathrm{H}$ & -0.2469701645 & 1.8147474442 & 2.8330518687 \\
\hline $\mathrm{H}$ & 0.8971688448 & 3.2054795208 & 0.8508030695 \\
\hline $\mathrm{H}$ & -1.3028525677 & 1.7829904509 & 1.3797783649 \\
\hline $\mathrm{H}$ & 2.4078753609 & 2.0363720881 & 2.4568488361 \\
\hline $\mathrm{H}$ & 1.4978694114 & 1.3716505610 & -1.0748391353 \\
\hline $\mathrm{H}$ & -0.9393510804 & -0.1952593427 & -0.4200460589 \\
\hline $\mathrm{H}$ & 0.7166075011 & -0.8261984935 & -0.4055799655 \\
\hline $\mathrm{H}$ & -0.1804543423 & 1.9562550971 & -1.0242151773 \\
\hline $\mathrm{H}$ & 2.9464422731 & 1.4039944503 & 0.8845090857 \\
\hline $\mathrm{H}$ & -2.9566552403 & -3.0933906147 & 1.1017905545 \\
\hline $\mathrm{H}$ & -2.7350760057 & -5.4239084896 & 0.3122402832 \\
\hline 11 & -0.4750734780 & -6.5793179718 & 0.367633590 \\
\hline
\end{tabular}




$\begin{array}{rrrr}\mathrm{H} & 1.5264674650 & -5.2925593486 & 1.1689992661 \\ \mathrm{H} & 1.3200678607 & -2.9463734176 & 1.9025064075 \\ \mathrm{H} & -2.7485236808 & -0.2261549492 & 4.5314866762 \\ \mathrm{H} & -2.5844637406 & -0.4674916933 & 6.9823774093 \\ \mathrm{H} & -0.6005274808 & -1.6596836947 & 8.0217212420 \\ \mathrm{H} & 1.2133509109 & -2.5366114189 & 6.5144402570 \\ \mathrm{H} & 1.0789353322 & -2.2569418524 & 4.0707223032 \\ \mathrm{H} & 1.6166002398 & -0.0922477184 & 3.0881014482 \\ \mathrm{H} & 2.1389398415 & -0.7808047294 & 1.5332622130\end{array}$

\section{Adamantan-1-yl-diphenyl-phosphane (9)}

UBPW91/6-31+G*, -1194.65809818968

xyz coordinates:

\begin{tabular}{|c|c|}
\hline $\mathrm{C}$ & 0.5934798586 \\
\hline $\mathrm{C}$ & 0.1706967457 \\
\hline $\mathrm{C}$ & 1.1673509906 \\
\hline $\mathrm{C}$ & 2.5347481595 \\
\hline $\mathrm{C}$ & 2.9357744730 \\
\hline $\mathrm{C}$ & 1.960413132 \\
\hline $\mathrm{P}$ & -1.575648093 \\
\hline $\mathrm{C}$ & -2.312923536 \\
\hline $\mathrm{C}$ & -3.425436512 \\
\hline $\mathrm{C}$ & -4.027253331 \\
\hline $\mathrm{C}$ & -3.51245705 \\
\hline C & -2.3974217090 \\
\hline $\mathrm{C}$ & -1.806462684 \\
\hline $\mathrm{C}$ & -2.5644366621 \\
\hline $\mathrm{C}$ & -1.766458521 \\
\hline $\mathrm{C}$ & -2.597219472 \\
\hline $\mathrm{C}$ & -2.9185138965 \\
\hline $\mathrm{C}$ & -3.7345975032 \\
\hline $\mathrm{C}$ & -2.905257744 \\
\hline $\mathrm{C}$ & -5.0522504360 \\
\hline $\mathrm{C}$ & -4.7291490091 \\
\hline $\mathrm{C}$ & -3.9154723029 \\
\hline $\mathrm{C}$ & -3.897644856 \\
\hline $\mathrm{H}$ & -3.9583067993 \\
\hline $\mathrm{H}$ & -5.6525665954 \\
\hline $\mathrm{H}$ & -5.6614651308 \\
\hline $\mathrm{H}$ & -5.6670059683 \\
\hline $\mathrm{H}$ & -3.6978769846 \\
\hline $\mathrm{H}$ & -4.5045473710 \\
\hline $\mathrm{H}$ & -2.0058377938 \\
\hline $\mathrm{H}$ & -1.9838031762 \\
\hline $\mathrm{H}$ & -3.4736910785 \\
\hline $\mathrm{H}$ & -0.8095795908 \\
\hline $\mathrm{H}$ & -1.518589398 \\
\hline $\mathrm{H}$ & -4.4895108458 \\
\hline $\mathrm{H}$ & -3.6806850006 \\
\hline $\mathrm{H}$ & -3.8116208929 \\
\hline $\mathrm{H}$ & -4.8863942395 \\
\hline $\mathrm{H}$ & -3.9679307269 \\
\hline $\mathrm{H}$ & -1.9817188863 \\
\hline 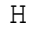 & -0.9341283216 \\
\hline$I$ & 0.86728887 \\
\hline & 3. \\
\hline
\end{tabular}

$\begin{array}{rr}0.2206231605 & 0.0125989669 \\ 0.0341924434 & 1.3500380902 \\ -0.1071208127 & 2.3461464085 \\ -0.0576745736 & 2.0252251305 \\ 0.1249470086 & 0.6914174799 \\ 0.2620085722 & -0.3122390503 \\ -0.0829247701 & 1.9935913910 \\ 1.6048675958 & 1.7138801020 \\ 1.9426641994 & 2.5234119653 \\ 3.2089685228 & 2.4377552271 \\ 4.1760214473 & 1.5568422114 \\ 3.8616259479 & 0.7607566373 \\ 2.5879158013 & 0.8350673424 \\ -1.2574783926 & 0.8368169972 \\ -2.5833398005 & 0.7086226321 \\ -3.6309407228 & -0.0746167562 \\ -3.0825739640 & -1.4835898600 \\ -1.7757540766 & -1.3575872088 \\ -0.7237904582 & -0.5754690200 \\ -2.0624471620 & -0.6042686748 \\ -2.6097553175 & 0.8034836770 \\ -3.9169626778 & 0.6791174984 \\ -1.5614720324 & 1.5825601127 \\ -1.3762578431 & -2.3641751121 \\ -1.1361133939 & -0.5217707674 \\ -2.7948731997 & -1.1677679278 \\ -2.8022561050 & 1.3567608332 \\ -4.3257479611 & 1.6846228965 \\ -4.6824318002 & 0.1385035707 \\ -4.5613014659 & -0.1607714717 \\ -0.5012833110 & -1.1408258029 \\ 0.2203667777 & -0.4944189334 \\ -2.4020606898 & 0.1873175318 \\ -2.9777057766 & 1.7131911474 \\ -0.6348060445 & 1.6980999673 \\ -1.9391948055 & 2.5994046397 \\ 1.2173257682 & 3.2484481796 \\ 3.4449183745 & 3.0754032773 \\ 5.1704610337 & 1.5009360252 \\ 4.6120399491 & 0.0791639585 \\ 2.3705663393 & 0.2125809446 \\ -0.2622284868 & 3.3888533759 \\ -0.1685761345 & 2.8177669293\end{array}$




$\begin{array}{rrrr}\mathrm{H} & 3.9999659806 & 0.1584845238 & 0.4344587517 \\ \mathrm{H} & 2.2627616579 & 0.4043190728 & -1.3556401787 \\ \mathrm{H} & -0.1364421771 & 0.3344144746 & -0.7926633689 \\ \mathrm{H} & -1.9803448608 & -2.8923434543 & -2.0392074705 \\ \mathrm{H} & -3.4907362095 & -3.8334370859 & -2.0612469029\end{array}$

\section{Radical Anion of Adamantan-1-yl-diphenyl-phosphane (9·-)}

\begin{tabular}{|c|c|c|c|}
\hline C & 0.5860284517 & 0.3975072943 & 0.0625293608 \\
\hline $\mathrm{C}$ & 0.1150645464 & 0.0010681788 & 1.3649190920 \\
\hline $\mathrm{C}$ & 1.1221765638 & -0.2345695065 & 2.3524413291 \\
\hline $\mathrm{C}$ & 2.4862940052 & -0.0836167302 & 2.0898485008 \\
\hline $\mathrm{C}$ & 2.9281037197 & 0.3235396524 & 0.7961355917 \\
\hline $\mathrm{C}$ & 1.9555755163 & 0.5548154914 & -0.1997949753 \\
\hline $\mathrm{P}$ & -1.6198562089 & -0.0945241097 & 1.9509378163 \\
\hline $\mathrm{C}$ & -2.3824554470 & 1.5618298891 & 1.7007424273 \\
\hline $\mathrm{C}$ & -3.0786068243 & 2.1278342828 & 2.8174780762 \\
\hline $\mathrm{C}$ & -3.5533222271 & 3.4420164781 & 2.8174590816 \\
\hline $\mathrm{C}$ & -3.3446646444 & 4.2838040606 & 1.6888589270 \\
\hline $\mathrm{C}$ & -2.6623223078 & 3.7481451303 & 0.5714085311 \\
\hline C & -2.2060271705 & 2.4234763385 & 0.5617236612 \\
\hline C & -2.5783734819 & -1.3059208453 & 0.7826326343 \\
\hline C & -1.8144782327 & -2.6519115247 & 0.7494011780 \\
\hline $\mathrm{C}$ & -2.6255381906 & -3.7180684606 & -0.0277941270 \\
\hline $\mathrm{C}$ & -2.8603884443 & -3.2310990815 & -1.4761083162 \\
\hline $\mathrm{C}$ & -3.6434996416 & -1.8988062950 & -1.4532580689 \\
\hline $\mathrm{C}$ & -2.8271220839 & -0.8376844647 & -0.6705391661 \\
\hline $\mathrm{C}$ & -5.0072434370 & -2.1178878179 & -0.7616362915 \\
\hline $\mathrm{C}$ & -4.7752232256 & -2.6046825586 & 0.6858253018 \\
\hline $\mathrm{C}$ & -3.9900839650 & -3.9359794911 & 0.6649743898 \\
\hline $\mathrm{C}$ & -3.9551813636 & -1.5460450668 & 1.4597512045 \\
\hline $\mathrm{H}$ & -3.8037253339 & -1.5429560080 & -2.4887404356 \\
\hline $\mathrm{H}$ & -5.5851479949 & -1.1736059106 & -0.7545711179 \\
\hline $\mathrm{H}$ & -5.6068391901 & -2.8601463423 & -1.3235978801 \\
\hline $\mathrm{H}$ & -5.7480550679 & -2.7525963701 & 1.1920993634 \\
\hline $\mathrm{H}$ & -3.8361782788 & -4.2999298225 & 1.6992715016 \\
\hline $\mathrm{H}$ & -4.5694411790 & -4.7143390083 & 0.1313994238 \\
\hline $\mathrm{H}$ & -2.0591057730 & -4.6685966340 & -0.0410930232 \\
\hline $\mathrm{H}$ & -1.8657774502 & -0.6687171601 & -1.1871223418 \\
\hline $\mathrm{H}$ & -3.3699879185 & 0.1247139891 & -0.6640037408 \\
\hline $\mathrm{H}$ & -0.8258585591 & -2.5066753485 & 0.2776897809 \\
\hline $\mathrm{H}$ & -1.6263495968 & -3.0059669628 & 1.7817043150 \\
\hline $\mathrm{H}$ & -4.5159945728 & -0.5941297168 & 1.5127841959 \\
\hline $\mathrm{H}$ & -3.8031835593 & -1.8873996454 & 2.5013647898 \\
\hline $\mathrm{H}$ & -3.2355472160 & 1.5088119415 & 3.7091391205 \\
\hline $\mathrm{H}$ & -4.0923319724 & 3.8211256280 & 3.6945066771 \\
\hline $\mathrm{H}$ & -3.7073747037 & 5.3169517555 & 1.6828152079 \\
\hline $\mathrm{H}$ & -2.4963282969 & 4.3766180815 & -0.3128280302 \\
\hline $\mathrm{H}$ & -1.6916056214 & 2.0525574792 & -0.3277159587 \\
\hline $\mathrm{H}$ & 0.8075460473 & -0.5409829696 & 3.3577816508 \\
\hline $\mathrm{H}$ & 3.2163442921 & -0.2873790040 & 2.8827140989 \\
\hline $\mathrm{H}$ & 3.9942845425 & 0.4477472493 & 0.5793315590 \\
\hline $\mathrm{H}$ & 2.2732020476 & 0.8588431811 & -1.2057044905 \\
\hline $\mathrm{H}$ & -0.1224900603 & 0.5654442241 & -0.7522749173 \\
\hline $\mathrm{H}$ & -1.8901016143 & -3.0909033562 & -1.9900161744 \\
\hline $\mathrm{H}$ & -3.4226113292 & -3.9945790354 & -2.0480903496 \\
\hline
\end{tabular}




\title{
5-Diphenylphosphanyl-adamantan-2-one (10)
}

\author{
UBPW91/6-31+G*, -1268.68665310129
}

xyz coordinates:

\begin{tabular}{lrrr}
$\mathrm{C}$ & 0.5108579294 & 0.1409885983 & 0.0753342268 \\
$\mathrm{C}$ & 0.0264883294 & 0.1282638347 & 1.5138273696 \\
$\mathrm{C}$ & 1.2759210996 & 0.1821209157 & 2.4393455352 \\
$\mathrm{C}$ & 2.1865999491 & -1.0261315181 & 2.1391288069 \\
$\mathrm{C}$ & 2.6546610946 & -0.9686024979 & 0.6709746209 \\
$\mathrm{C}$ & 1.4156343148 & -1.0275308518 & -0.2673924885 \\
$\mathrm{C}$ & -0.7536510221 & -1.1987974056 & 1.7595428787 \\
$\mathrm{C}$ & 0.1551584321 & -2.4169665666 & 1.4584979455 \\
$\mathrm{C}$ & 1.4046289421 & -2.3383185669 & 2.3856634633 \\
$\mathrm{C}$ & 0.6264541591 & -2.3496443367 & -0.0123140172 \\
$\mathrm{P}$ & -0.7763117549 & -4.0160665862 & 1.9859166073 \\
$\mathrm{O}$ & 0.2038925440 & 1.0255939473 & -0.7375518593 \\
$\mathrm{H}$ & 1.7158535249 & -0.9610198518 & -1.3261020791 \\
$\mathrm{H}$ & 3.3186438842 & -1.8209519451 & 0.4367994146 \\
$\mathrm{H}$ & 3.2307241228 & -0.0447943184 & 0.4809275231 \\
$\mathrm{H}$ & 3.0657044207 & -1.0012300074 & 2.8082074158 \\
$\mathrm{H}$ & 0.9384205499 & 0.1689539851 & 3.4921489038 \\
$\mathrm{H}$ & 1.8218616544 & 1.1304924927 & 2.2834999184 \\
$\mathrm{H}$ & -0.6333146406 & 0.9943921288 & 1.6862338325 \\
$\mathrm{H}$ & -0.2270449015 & -2.3976794216 & -0.7092822686 \\
$\mathrm{H}$ & 1.2871638818 & -3.2011642948 & -0.2507977253 \\
$\mathrm{H}$ & -1.6558597304 & -1.2187767540 & 1.1246432500 \\
$\mathrm{H}$ & -1.0970833178 & -1.2116787058 & 2.8108242185 \\
$\mathrm{H}$ & 2.0704068354 & -3.2010409690 & 2.2025170632 \\
$\mathrm{H}$ & 1.0877687600 & -2.3884146799 & 3.4434248916 \\
$\mathrm{C}$ & -2.4936850881 & -3.9744637098 & 1.2632674334 \\
$\mathrm{C}$ & 0.0958042142 & -5.3780004892 & 1.0610957817 \\
$\mathrm{C}$ & 1.2069871940 & -5.9684325274 & 1.7107728549 \\
$\mathrm{C}$ & 1.9112606542 & -7.0294481437 & 1.1177315973 \\
$\mathrm{C}$ & 1.5016956109 & -7.5382768521 & -0.1267686120 \\
$\mathrm{C}$ & 0.3879956381 & -6.9747566774 & -0.7740205562 \\
$\mathrm{C}$ & -0.3060836106 & -5.9017089818 & -0.1880981400 \\
$\mathrm{H}$ & 1.5125630174 & -5.6121846457 & 2.7009321942 \\
$\mathrm{H}$ & 2.7681862251 & -7.4699703002 & 1.6390216174 \\
$\mathrm{H}$ & 2.0386474887 & -8.3766477037 & -0.5832236592 \\
$\mathrm{H}$ & 0.0550946801 & -7.3732019595 & -1.7386313363 \\
$\mathrm{H}$ & -4.5235717267 & -3.2299087791 & -1.4113551298 \\
$\mathrm{H}$ & -1.1741307237 & -5.4833549987 & -0.7054217041 \\
$\mathrm{C}$ & -3.5017256294 & -4.4183712751 & 2.1549369466 \\
$\mathrm{C}$ & -4.8532051088 & -4.4513241364 & 1.7712239425 \\
$\mathrm{C}$ & -5.2273183145 & -4.0224405283 & 0.4863357635 \\
$\mathrm{C}$ & -4.2412437390 & -3.5682154164 & -0.4080305265 \\
$\mathrm{C}$ & -2.8883446391 & -3.5483932929 & -0.0268456427 \\
$\mathrm{H}$ & -3.2243848779 & -4.7388717417 & 3.1660562793 \\
$\mathrm{H}$ & -5.6114333821 & -4.8031301625 & 2.4806976051 \\
\hline
\end{tabular}




\section{Radical Anion of 5-Diphenylphosphanyl-adamantan-2-one (10--)}

UBPW91/6-31+G*, -1268.74945147265

xyz coordinates:

\begin{tabular}{|c|c|c|c|}
\hline C & -0.0511976575 & 0.0523005627 & 0.0829746500 \\
\hline C & 0.0478967565 & -0.0025449467 & 1.5925309215 \\
\hline C & 1.5502471481 & -0.0502935636 & 1.9930591726 \\
\hline C & 2.2103885575 & -1.2866781966 & 1.34412657 \\
\hline $\mathrm{C}$ & 2.0903346723 & -1.1973367260 & -0.192461573 \\
\hline $\mathrm{C}$ & 0.5870452800 & -1.1395089983 & -0.588053168 \\
\hline $\mathrm{C}$ & -0.6508671340 & -1.3065614825 & 2.09487669 \\
\hline $\mathrm{C}$ & 0.0051361773 & -2.5492894836 & 1.45592739 \\
\hline $\mathrm{C}$ & 1.5040070662 & -2.5712229617 & 1.84241697 \\
\hline $\mathrm{C}$ & -0.1184446644 & -2.4441113950 & -0.07420067 \\
\hline $\mathrm{P}$ & -0.8118902948 & -4.1134601936 & 2.28845237 \\
\hline 0 & -0.6086544860 & 1.0032787821 & -0.54918402 \\
\hline $\mathrm{H}$ & 0.4733060156 & -1.0576629265 & -1.68303374 \\
\hline $\mathrm{H}$ & 2.5637066206 & -2.0788176829 & -0.66595782 \\
\hline $\mathrm{H}$ & 2.6148197291 & -0.3012129817 & -0.57223221 \\
\hline $\mathrm{H}$ & 3.2780729721 & -1.3271552704 & 1.6293565 \\
\hline $\mathrm{H}$ & 1.6379890668 & -0.1013370419 & 3.095721 \\
\hline $\mathrm{H}$ & 2.0572995005 & 0.8767742430 & 1.6675208 \\
\hline $\mathrm{H}$ & -0.4462536224 & 0.8822267357 & 2.0 \\
\hline $\mathrm{H}$ & -1.1793434815 & -2.4266812046 & -0.3 \\
\hline $\mathrm{H}$ & 0.3563450 & -3.3122052573 & -0.5 \\
\hline $\mathrm{H}$ & -1.7268877650 & -1.2708594455 & 1.84684198 \\
\hline $\mathrm{H}$ & -0.5726488405 & -1.3492473065 & 3.19758099 \\
\hline $\mathrm{H}$ & 1.9926870653 & -3.4619136375 & 1.40769579 \\
\hline $\mathrm{H}$ & 1.6074887298 & -2.6483320728 & 2.94143248 \\
\hline $\mathrm{C}$ & -2.5931241517 & -4.0745228588 & 1.80672079 \\
\hline $\mathrm{C}$ & -0.0263145347 & -5.5734779272 & 1.49240964 \\
\hline $\mathrm{C}$ & 0.7342414462 & -6.4099801823 & 2.36087575 \\
\hline $\mathrm{C}$ & 1.3610288146 & -7.5778152159 & 1.912093493 \\
\hline $\mathrm{C}$ & 1.2452259145 & -7.9718649936 & $0.55240577 \varepsilon$ \\
\hline $\mathrm{C}$ & 0.5053332286 & -7.1584079971 & -0.327418663 \\
\hline $\mathrm{C}$ & -0.1123827374 & -5.9775215816 & 0.119441575 \\
\hline $\mathrm{H}$ & 0.8252854038 & -6.1299372791 & 3.417307516 \\
\hline $\mathrm{H}$ & 1.9392683061 & -8.1869919482 & 2.61686776 \\
\hline $\mathrm{H}$ & 1.7239458346 & -8.8893394023 & 0.194270963 \\
\hline $\mathrm{H}$ & 0.4102294204 & -7.4453900957 & -1.382008302 \\
\hline $\mathrm{H}$ & -0.6655653641 & -5.3743223718 & -0.60367125 \\
\hline $\mathrm{C}$ & -3.5096300572 & -3.8428431252 & 2.875658472 \\
\hline $\mathrm{C}$ & -4.8964140554 & -3.8648365888 & 2.686758129 \\
\hline $\mathrm{C}$ & -5.4373781747 & -4.1341974887 & 1.404798951 \\
\hline $\mathrm{C}$ & -4.5522292776 & -4.3513101990 & 0.32920822 \\
\hline $\mathrm{C}$ & -3.1608540180 & -4.3099275512 & $0.51441975^{\circ}$ \\
\hline $\mathrm{H}$ & -3.1139038398 & -3.6518012363 & $3.88040514]$ \\
\hline $\mathrm{H}$ & -5.5614778794 & -3.6778744435 & 3.53834878 \\
\hline $\mathrm{H}$ & -6.5210890879 & -4.1686379387 & 1.25107778 \\
\hline $\mathrm{H}$ & -4.9530811593 & -4.5504921113 & -0.672245850 \\
\hline $\mathrm{H}$ & -2.5169364251 & -4.4698056305 & -0.3531388 \\
\hline
\end{tabular}

\section{5-Trimehylstannyl-adamantan-2-one (11)}

UBPW91/6-31+G*， -587.1947165499

xyz coordinates: 


$\begin{array}{lrrr}\mathrm{C} & -0.5161496484 & 0.0023502239 & -0.1809390290 \\ \mathrm{C} & -0.5115792039 & 0.0008761234 & 1.3625693917 \\ \mathrm{C} & 0.9508589736 & 0.0042625328 & 1.8795265449 \\ \mathrm{C} & 1.7050441484 & 1.2650083701 & 1.3787454948 \\ \mathrm{C} & 1.6896769015 & 1.2748194384 & -0.1678109328 \\ \mathrm{C} & 0.2183675355 & 1.2708921086 & -0.7028332652 \\ \mathrm{C} & -1.2468061965 & 1.2568201442 & 1.8768575437 \\ \mathrm{C} & -0.5165747753 & 2.5329774575 & 1.3678458595 \\ \mathrm{C} & 0.9618915077 & 2.5244949262 & 1.8827928956 \\ \mathrm{C} & -0.4794675378 & 2.4939069475 & -0.1455550024 \\ \mathrm{Sn} & 3.7962386778 & 1.2694621493 & 2.1191310621 \\ \mathrm{O} & -0.9599386112 & 3.3878786940 & -0.8610868046 \\ \mathrm{H} & -1.0366626483 & 3.4479179662 & 1.6971587316 \\ \mathrm{H} & -1.2666175090 & 1.2753778993 & 2.9826272035 \\ \mathrm{H} & -2.2975699601 & 1.2612589949 & 1.5329927232 \\ \mathrm{H} & -1.0306545005 & -0.9034153816 & 1.7297569507 \\ \mathrm{H} & -0.0026237032 & -0.8941185897 & -0.5760991055 \\ \mathrm{H} & -1.5517503337 & -0.0195605649 & -0.5674697744 \\ \mathrm{H} & 0.2061963355 & 1.3135904679 & -1.8046955124 \\ \mathrm{H} & 1.4672614899 & 3.4477639061 & 1.5432877523 \\ \mathrm{H} & 0.9336649710 & 2.5607548503 & 2.9881404676 \\ \mathrm{H} & 2.2157389510 & 2.1626761467 & -0.5653487553 \\ \mathrm{H} & 2.2010267972 & 0.3854217236 & -0.5818600454 \\ \mathrm{H} & 0.9455551060 & -0.0282391751 & 2.9857918475 \\ \mathrm{H} & 1.4614511498 & -0.9141666938 & 1.5321507176 \\ \mathrm{C} & 4.8185654226 & 3.0444677036 & 1.4006765732 \\ \mathrm{C} & 3.7772753502 & 1.2694215553 & 4.2884142095 \\ \mathrm{C} & 4.8099663892 & -0.5049620475 & 1.3913387034 \\ \mathrm{H} & 4.8082483831 & 1.2664502451 & 4.6795190739 \\ \mathrm{H} & 3.2627232757 & 2.1660393381 & 4.6710751017 \\ \mathrm{H} & 3.2558632412 & 0.3775117728 & 4.6726862187 \\ \mathrm{H} & 5.8508219994 & -0.5267900863 & 1.7545322649 \\ \mathrm{H} & 4.2986521167 & -1.4158511929 & 1.7435597638 \\ \mathrm{H} & 4.8258612631 & -0.5177364104 & 0.2891337127 \\ \mathrm{H} & 5.8565705756 & 3.0601490067 & 1.7730385096 \\ \mathrm{H} & 4.8439617207 & 3.0629917526 & 0.2988966098 \\ \mathrm{H} & 4.3095579812 & 3.9566541002 & 1.7528812345 \\ & & & \\ & & & \end{array}$

\section{Radical Anion 5-Trimehylstannyl-adamantan-2-one (11•-)}

UBPW91/6-31+G*, -587.2520955043

xyz coordinates:

$\begin{array}{lr}\mathrm{C} & -0.5493256192 \\ \mathrm{C} & -0.5524541895 \\ \mathrm{C} & 0.9121398067 \\ \mathrm{C} & 1.6665076212 \\ \mathrm{C} & 1.6513464785 \\ \mathrm{C} & 0.1800380776 \\ \mathrm{C} & -1.2775110063 \\ \mathrm{C} & -0.5484792242 \\ \mathrm{C} & 0.9195159310 \\ \mathrm{C} & -0.4894769684 \\ \mathrm{Sn} & 3.7476004439 \\ \mathrm{O} & -0.1890605904 \\ \mathrm{H} & -1.0636813266 \\ \mathrm{H} & -1.3077611162 \\ \mathrm{H} & -2.3253661584 \\ \mathrm{H} & -1.0710157085\end{array}$

-0.1662462950
1.3780490435
1.8929335898
1.3895533638
-0.1611584897
-0.6778849291
1.8952181601
1.3804189075
1.9069458919
-0.1466084618
2.1010810742
-0.7466367077
1.7404758542
3.0031945702
1.5371317374
1.7491143753 


$\begin{array}{rrrr}\mathrm{H} & -0.0556914215 & -0.9590188382 & -0.5456627351 \\ \mathrm{H} & -1.5890306023 & -0.0461008272 & -0.5474138662 \\ \mathrm{H} & 0.1835211862 & 1.2290278722 & -1.7851393385 \\ \mathrm{H} & 1.4183218372 & 3.3994446295 & 1.5497438207 \\ \mathrm{H} & 0.9350079989 & 2.5094480210 & 3.0162228747 \\ \mathrm{H} & 2.1649991063 & 2.1075326883 & -0.5584686658 \\ \mathrm{H} & 2.1961686047 & 0.3254445038 & -0.5479844654 \\ \mathrm{H} & 0.9076198758 & -0.0676927670 & 3.0003236259 \\ \mathrm{H} & 1.4221953958 & -0.9577436762 & 1.5446826637 \\ \mathrm{C} & 4.7562741657 & 3.0570074786 & 1.3801385001 \\ \mathrm{C} & 3.7460615799 & 1.3240969806 & 4.2737406056 \\ \mathrm{C} & 4.8008057819 & -0.4658647517 & 1.3310289481 \\ \mathrm{H} & 4.7578952892 & 1.1639764837 & 4.6804845990 \\ \mathrm{H} & 3.3829664107 & 2.3066092556 & 4.6182843007 \\ \mathrm{H} & 3.0762619423 & 0.5491301932 & 4.6811804033 \\ \mathrm{H} & 5.7898764392 & -0.5786111474 & 1.8037244361 \\ \mathrm{H} & 4.2193102907 & -1.3848847144 & 1.5102529952 \\ \mathrm{H} & 4.9439134163 & -0.3575644716 & 0.2431448084 \\ \mathrm{H} & 5.7976535402 & 3.0832529083 & 1.7425560420 \\ \mathrm{H} & 4.7703049709 & 3.0772766538 & 0.2778904692 \\ \mathrm{H} & 4.2410254703 & 3.9651349653 & 1.7342551424\end{array}$




\section{GAS PHASE EXAMPLES}

\section{Chloro-2-Oxo-Adamantane (2c)}

\section{Neutral}

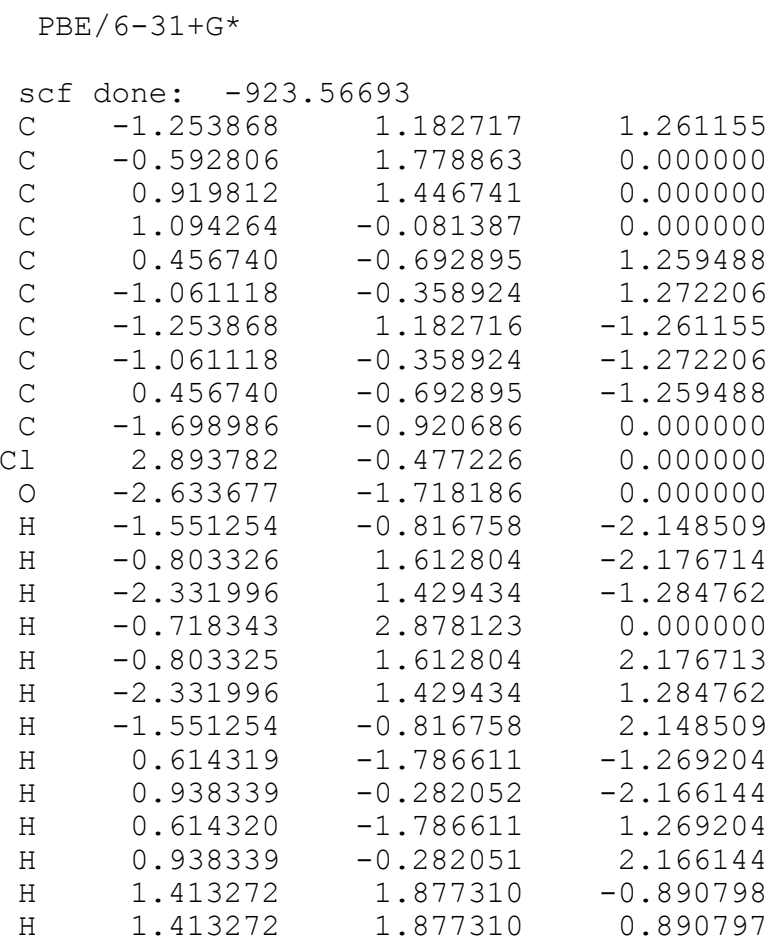

\section{Radical}

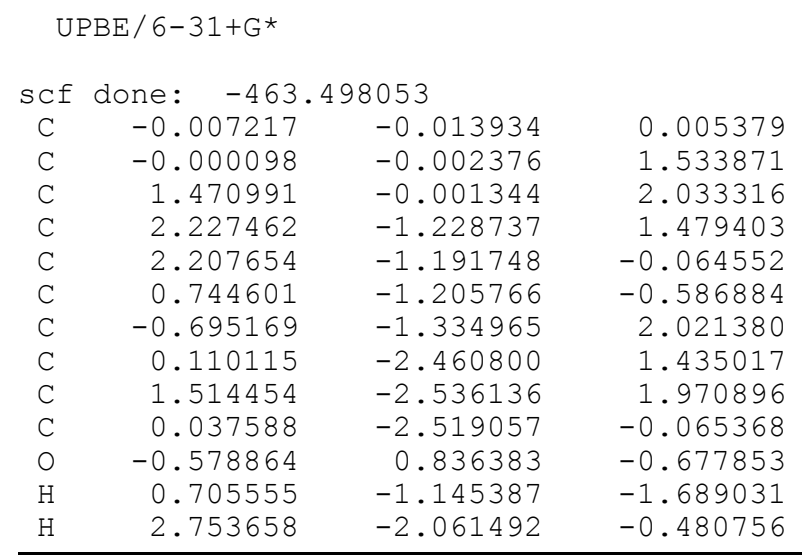




$\begin{array}{rrrr}\mathrm{H} & 2.725938 & -0.284670 & -0.430345 \\ \mathrm{H} & 3.274266 & -1.218098 & 1.840950 \\ \mathrm{H} & 1.481776 & -0.006202 & 3.141315 \\ \mathrm{H} & 1.973201 & 0.931708 & 1.713299 \\ \mathrm{H} & -0.551390 & 0.885763 & 1.890499 \\ \mathrm{H} & -1.003900 & -2.551457 & -0.435757 \\ \mathrm{H} & 0.573536 & -3.395019 & -0.478151 \\ \mathrm{H} & -1.747861 & -1.349259 & 1.682898 \\ \mathrm{H} & -0.692777 & -1.348730 & 3.128061 \\ \mathrm{H} & 2.057364 & -3.421471 & 1.587931 \\ \mathrm{H} & 1.534524 & -2.576591 & 3.076877\end{array}$

\section{RA}

$\mathrm{UPBE} / 6-31+\mathrm{G}^{*}$

scf done: -923.576738

$\begin{array}{rrrr}\mathrm{C} & -1.186369 & -1.435998 & -1.084036 \\ \mathrm{C} & -1.156398 & -1.433298 & 0.461532 \\ \mathrm{C} & 0.328188 & -1.375395 & 0.940763 \\ \mathrm{C} & 0.913097 & -0.108380 & 0.381346 \\ \mathrm{C} & 0.973709 & -0.096741 & -1.112764 \\ \mathrm{C} & -0.527524 & -0.140225 & -1.636628 \\ \mathrm{C} & -1.904724 & -0.188386 & 0.990573 \\ \mathrm{C} & -1.246571 & 1.108591 & 0.439981 \\ \mathrm{C} & 0.268777 & 1.127560 & 0.923081 \\ \mathrm{C} & -1.256163 & 1.070744 & -1.078876 \\ \mathrm{Cl} & 3.484242 & -0.083823 & 1.256863 \\ \mathrm{O} & -1.774428 & 1.953063 & -1.788932 \\ \mathrm{H} & -1.790905 & 2.010494 & 0.782234 \\ \mathrm{H} & -1.875075 & -0.172977 & 2.100963 \\ \mathrm{H} & -2.973051 & -0.223275 & 0.696928 \\ \mathrm{H} & -1.648160 & -2.353685 & 0.844749 \\ \mathrm{H} & -0.638157 & -2.321213 & -1.471258 \\ \mathrm{H} & -2.228851 & -1.515775 & -1.452323 \\ \mathrm{H} & -0.570251 & -0.109495 & -2.743017 \\ \mathrm{H} & 0.765483 & 2.046545 & 0.559586 \\ \mathrm{H} & 0.284954 & 1.154021 & 2.029648 \\ \mathrm{H} & 1.478440 & 0.808307 & -1.499434 \\ \mathrm{H} & 1.511949 & -0.976982 & -1.513918 \\ \mathrm{H} & 0.373641 & -1.383684 & 2.047799 \\ \mathrm{H} & 0.881418 & -2.265572 & 0.581340 \\ & & & \end{array}$

\section{TS}

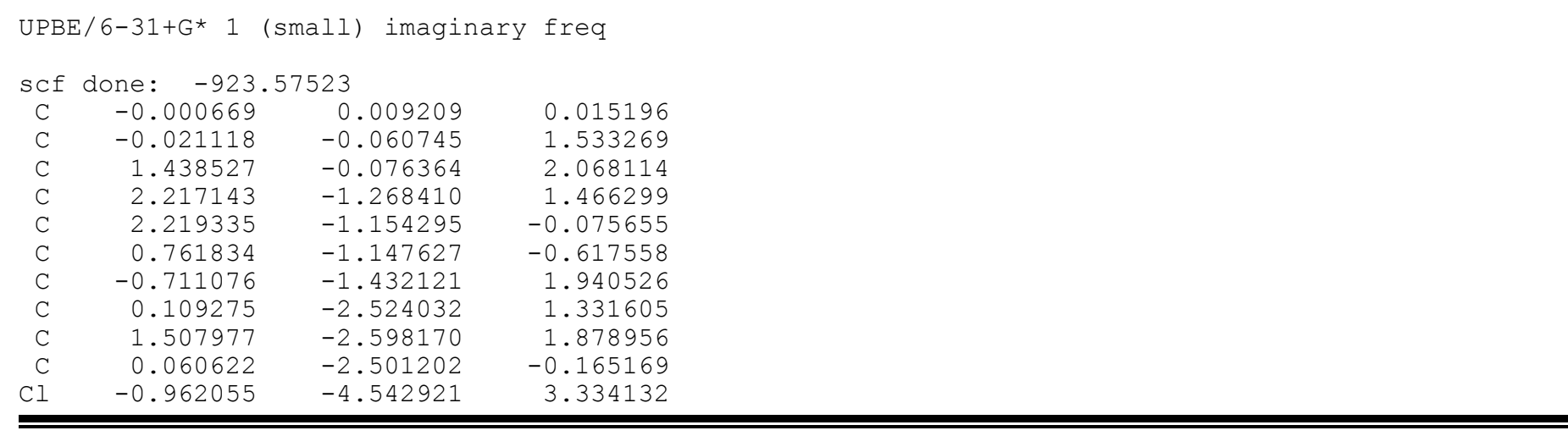




\begin{tabular}{lrrr}
$\mathrm{O}$ & -0.571477 & 0.892485 & -0.645566 \\
$\mathrm{H}$ & 0.742561 & -1.031584 & -1.718586 \\
$\mathrm{H}$ & 2.770087 & -2.009568 & -0.520461 \\
$\mathrm{H}$ & 2.743216 & -0.231631 & -0.397480 \\
$\mathrm{H}$ & 3.262345 & -1.257963 & 1.841611 \\
$\mathrm{H}$ & 1.418356 & -0.155521 & 3.174193 \\
$\mathrm{H}$ & 1.941452 & 0.880437 & 1.820433 \\
$\mathrm{H}$ & -0.583750 & 0.806704 & 1.927955 \\
$\mathrm{H}$ & -0.974394 & -2.530054 & -0.556007 \\
$\mathrm{H}$ & 0.619750 & -3.342844 & -0.620907 \\
$\mathrm{H}$ & -1.756916 & -1.448955 & 1.582030 \\
$\mathrm{H}$ & -0.736711 & -1.519630 & 3.041831 \\
$\mathrm{H}$ & 2.061445 & -3.464184 & 1.464627 \\
$\mathrm{H}$ & 1.488849 & -2.713096 & 2.977924 \\
\hline
\end{tabular}


PART 2. Supplementary Material 
Table S1. Summary of main results for the unsubstituted bridgehead alkyl halides ${ }^{a}$

\begin{tabular}{rrccc|}
\hline & 1a & 2a & 3a & 4a \\
\hline $\boldsymbol{D}$ & 90.52 & 85.81 & 86.76 & 79.44 \\
$\Delta E(\mathrm{RX}+\mathrm{e}->\mathrm{R} .+\mathrm{Cl}$ - , solution) & -70.29 & -75.00 & -74.06 & -81.37 \\
â (degrees) $^{b}$ & 107.5 & 113.1 & 112.8 & 118.8 \\
\%s character C. ${ }^{c}$ & 18.80 & 10.81 & 12.29 & 2.96 \\
spin(C. $^{d}$ & 0.910 & 0.866 & 0.893 & 0.877 \\
\hline
\end{tabular}

a) BPW91/6-31+G*, al energies in $\mathrm{kcal} / \mathrm{mol}$; see also Fig. 2 on main text

b) average $\mathrm{C}<\mathrm{Cipso}<\mathrm{C}<\mathrm{C}$ angle in the radical formed

c) hybridization of the natural orbital holding the lone electron in the radical

d) natural spin population on the radical center

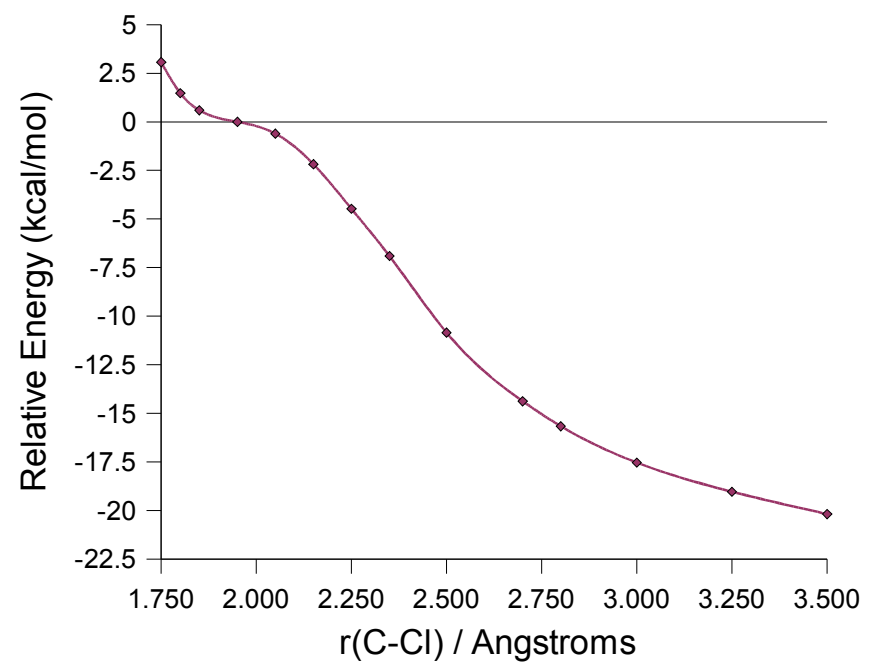

Figure $\boldsymbol{S} 1$ Quassi-dissociative anionic profile obtained for the 1-chloro-2-oxo-norbornane (1) $\mathbf{1 b}$; similar profiles have been obtained for other $\alpha$-oxo substituted derivatives and $\mathbf{4 b}$ 
Table S2. Solution phase energies

\begin{tabular}{|c|c|c|c|c|c|c|}
\hline $\mathrm{sp \epsilon}$ & & $\mathrm{E}$ (neutral) & $E(R A)$ & $\mathrm{E}$ (radical) & $\mathrm{E}(\mathrm{TS})$ & \multirow{2}{*}{$\begin{array}{c}\text { E(chloride) } \\
-460.38769\end{array}$} \\
\hline $1 \mathrm{a}$ & $\begin{array}{c}\mathrm{E}^{a} \\
\mathrm{ZPE}^{b}\end{array}$ & $\begin{array}{c}-733.53314 \\
102.608\end{array}$ & & $\begin{array}{c}-273.25390 \\
100.370\end{array}$ & & \\
\hline \multirow[t]{2}{*}{$1 \mathrm{~b}$} & $\mathrm{E}^{a}$ & -807.55779 & & -347.28089 & & \\
\hline & $\mathrm{ZPE}^{b}$ & 90.495 & & 88.357 & & \\
\hline \multirow[t]{2}{*}{$1 \mathrm{c}$} & $\mathrm{E}^{a}$ & -807.56095 & -807.63030 & -347.28558 & -807.62838 & \\
\hline & $\mathrm{ZPE}^{b}$ & 90.643 & 95.997 & 88.354 & 95.908 & \\
\hline \multirow[t]{2}{*}{$2 a$} & $\mathrm{E}^{a}$ & -850.27681 & & -390.00488 & & \\
\hline & $\mathrm{ZPE}^{b}$ & 142.985 & & 140.625 & & \\
\hline \multirow[t]{2}{*}{$2 b$} & $E^{a}$ & -924.29611 & & -464.02883 & & \\
\hline & $\mathrm{ZPE}^{b}$ & 131.160 & & 128.858 & & \\
\hline \multirow[t]{2}{*}{$2 c$} & $E^{a}$ & -924.30202 & -924.36750 & -464.03252 & -924.36280 & \\
\hline & $\mathrm{ZPE}^{b}$ & 131.500 & 136.225 & 129.028 & 135.281 & \\
\hline \multirow[t]{2}{*}{$3 a$} & $E^{a}$ & -772.84765 & & -312.57381 & & \\
\hline & $\mathrm{ZPE}^{b}$ & 120.621 & & 118.186 & & \\
\hline \multirow[t]{2}{*}{$3 b$} & $E^{a}$ & -846.87165 & -846.93448 & -386.60195 & -846.93349 & \\
\hline & $\mathrm{ZPE}^{b}$ & 108.553 & 106.003 & 106.204 & 105.950 & \\
\hline \multirow[t]{2}{*}{$3 c$} & $E^{a}$ & -846.87755 & -846.93930 & -386.60695 & -846.93767 & \\
\hline & $\mathrm{ZPE}^{b}$ & 114.095 & 105.986 & 106.277 & 105.895 & \\
\hline \multirow[t]{2}{*}{$4 a$} & $\mathrm{E}^{a}$ & -968.17409 & & -507.91154 & & \\
\hline & $\mathrm{ZPE}^{b}$ & 195.857 & & 193.010 & & \\
\hline \multirow[t]{2}{*}{$4 b$} & $E^{a}$ & -1042.20167 & & -581.94423 & & \\
\hline & $\mathrm{ZPE}^{b}$ & 191.807 & & 190.176 & & \\
\hline
\end{tabular}

a) total energies in Hartree; $b$ ) Zero point energy corrections in kcal/mol 

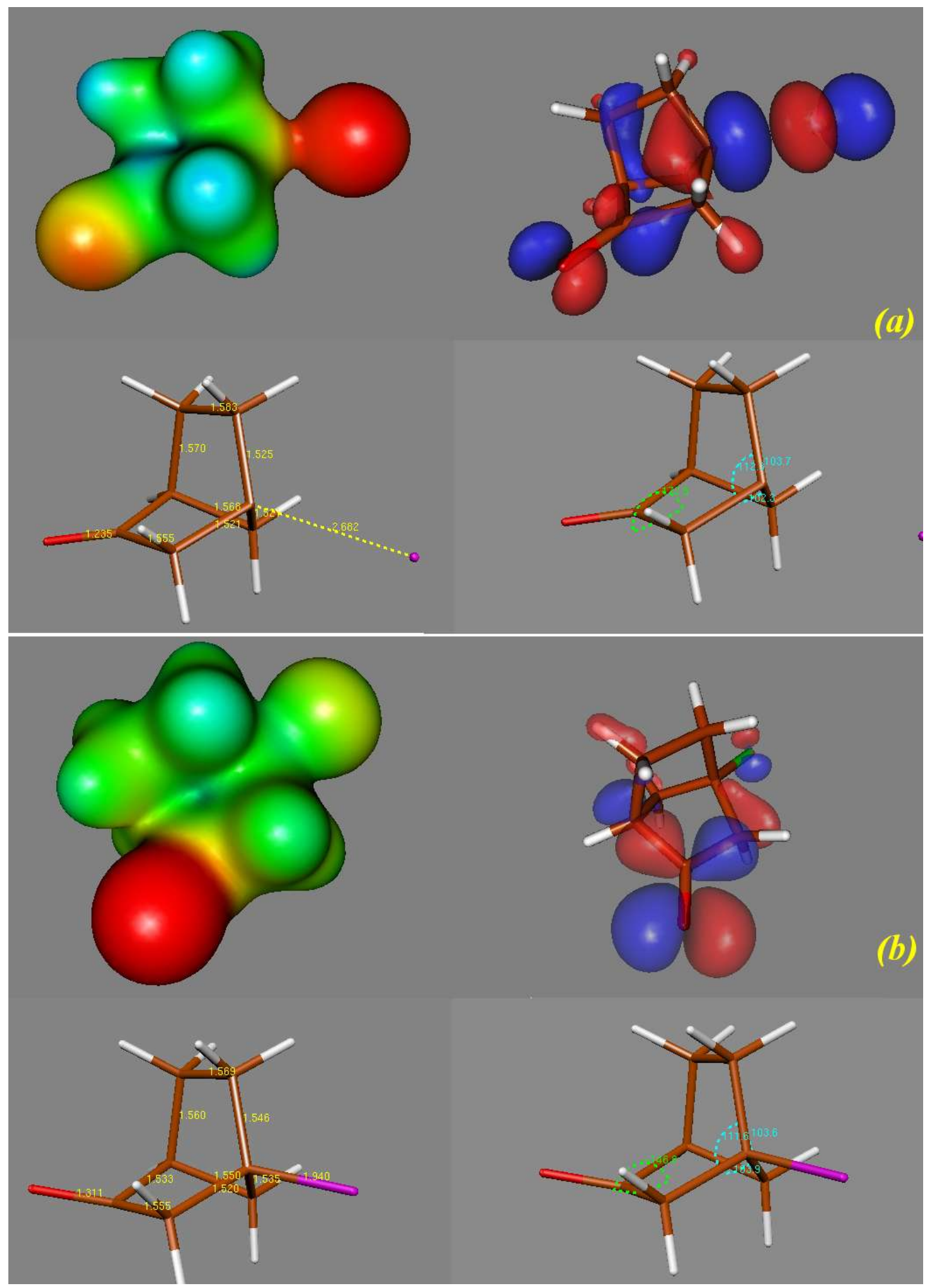

Figure S2. a) Gas phase BPW91/6-31+G* RA of 1c. top left: electrostatic potential, top right: SOMO diagram, bottom left: main distances and bottom right: main angles (cyan) and dehidral (green labels). b) The same for the solution phase RA of 1c. 
2B.Gas phase results obtained with different DFT methods.

Table S3. Details on the gas phase potential energy surface scan with diferent DFT methods and basis sets for $\mathbf{1 b}, \mathbf{1 c}, \mathbf{2 c}$ and $\mathbf{3 b} .^{a}$

\begin{tabular}{|c|c|c|c|c|c|c|c|c|c|c|c|c|c|c|c|c|}
\hline \multirow{3}{*}{\begin{tabular}{|l|} 
compound \\
level of theory \\
species \\
\end{tabular}} & \multicolumn{10}{|c|}{\begin{tabular}{|l|l|}
$1 \mathrm{~b}$ & $1 \mathrm{c}$ \\
\end{tabular}} & \multirow{2}{*}{\multicolumn{2}{|c|}{\begin{tabular}{|l|} 
2c \\
B3LYP/6-311+G(2d,p) \\
//B3LYP/6-31+G*
\end{tabular}}} & \multicolumn{4}{|l|}{$3 b$} \\
\hline & \multicolumn{2}{|c|}{ BPW91/6-31+G* } & \multicolumn{2}{|c|}{$\begin{array}{l}\text { B3LYP/6-311+G(2d,p) } \\
\text { //B3LYP/6-31+G* }\end{array}$} & \multicolumn{2}{|c|}{ BPW91/6-31+G* } & \multicolumn{2}{|l|}{$\mathrm{PBE} / 6-31+\mathrm{G}^{*}$} & \multicolumn{2}{|c|}{$\begin{array}{l}\text { B3LYP/6-311+G(2d,p) } \\
\text { //B3LYP/6-31+G* }\end{array}$} & & & \multicolumn{2}{|c|}{ BPW91/6-31+G* } & \multicolumn{2}{|c|}{$\begin{array}{l}\text { B3LYP/6-311+G(2d,p) } \\
\text { //B3LYP/6-31+G* }\end{array}$} \\
\hline & $E$ & ZPE & $\mathrm{E}$ & ZPE & $E$ & $\overline{\mathrm{ZPE}}$ & $E$ & ZPE & $\mathrm{E}$ & ZPE & $\mathrm{E}$ & ZPE & $\mathrm{E}$ & ZPE & $E$ & ZPE \\
\hline $\mathrm{RCl}$ & -807.54984 & 0.14463 & -807.71980 & 0.14835 & \begin{tabular}{|l|l|}
5 & -807.55356
\end{tabular} & 0.14470 & -806.96362 & 0.14447 & -807.72334 & 0.21388 & -924.50915 & 0.21388 & -846.86367 & 0.17350 & -847.05369 & 0.17787 \\
\hline $\mathrm{RCl} \cdot-$ (vertical) $^{b}$ & -807.53180 & & -807.69986 & & -807.53480 & & -806.94737 & & -807.70212 & & -924.49144 & & -846.84314 & & -847.03167 & \\
\hline $\mathrm{RCl} \cdot-$ & -807.55212 & 0.14060 & & & -807.55563 & 0.14019 & -806.96467 & 0.13988 & & & & & & & & \\
\hline TS: $\mathrm{RCl}^{-} \quad$-> R. + Cl- & -807.55155 & 0.14062 & & & -807.55553 & 0.14041 & -806.96430 & 0.14018 & & & & & & & & \\
\hline$(\mathrm{R} \cdot) \mathrm{Cl}^{-c}$ & & & & & -807.56092 & 0.14068 & -806.96961 & 0.14046 & & & & & -846.88164 & 0.16968 & & \\
\hline R. & -347.27409 & 0.14114 & -347.41022 & 0.14481 & \begin{tabular}{l|l}
-347.27987 \\
\end{tabular} & 0.14112 & -346.88722 & 0.14089 & -347.41538 & 0.21130 & -464.21108 & 0.21130 & -386.59521 & 0.16964 & -386.75164 & 0.17396 \\
\hline $\mathrm{Cl}-$ & -460.26746 & & -460.30373 & & -460.26746 & & -460.06500 & & -460.30373 & & -460.30373 & & -460.26746 & & -460.30373 & \\
\hline $\mathrm{Cl} \cdot$ & -460.13299 & & -460.16706 & & -460.13299 & & -459.92935 & & -460.16706 & & -460.16706 & & -460.13299 & & -460.16706 & \\
\hline calculated properties $^{d}$ & $e$ & $\bar{f}$ & & & & & & & & & & & & & & \\
\hline VEA /eV & -0.491 & & -0.543 & & -0.511 & & -0.442 & & -0.577 & & -0.482 & & -0.559 & & -0.599 & \\
\hline D-/ (kcal/mol) & 5.201 & 3.012 & 3.673 & 1.455 & 3.908 & 1.663 & 7.155 & 4.907 & 2.656 & 1.041 & -3.550 & -5.165 & 0.629 & -1.794 & -1.048 & -3.500 \\
\hline $\mathrm{D} /(\mathrm{kcal} / \mathrm{mol})$ & 89.585 & 87.397 & 89.436 & 87.218 & 88.292 & 86.047 & 92.274 & 90.026 & 88.419 & 88.402 & 82.213 & 80.598 & 85.013 & 82.590 & 84.715 & 82.263 \\
\hline $\mathrm{E}_{r e l}(\mathrm{RA}) /(\mathrm{kcal} / \mathrm{mol})$ & -6.632 & -6.971 & & & -5.205 & -5.792 & -7.819 & -8.453 & -5.488 & -5.813 & -5.488 & -5.488 & & & & \\
\hline$E_{a} /(\mathrm{kcal} / \mathrm{mol})$ & 0.355 & 0.366 & & & 0.062 & 0.201 & 0.236 & 0.425 & & & & & & & & \\
\hline
\end{tabular}

a) Energies of the stationary points found: total energies and zero point corrections in Hartrees

b) Ground state anion on the frozen coordinates of the neutral molecule

c) Some electrostatic complexes found at long C-Cl distances $(>3.4 \mathrm{~A})$,

d) Calculated properties (see main text Fig. 2); e) uncorrected and $f$ ) including zero corrections.

Table S4. Comparison of the vertical electron affinities obtained with different DFT methods.

\begin{tabular}{|lccccccc|}
\hline & 1b & 1c & 2b & 2c & 3b & 3c & 4b \\
\hline PBE/6-31+G & -0.04 & -0.05 & & -0.04 & -0.22 & & \\
BPW91/6-31+G* & -0.49 & -0.51 & -0.56 & -0.44 & -0.56 & -0.60 & -0.40 \\
B3LYP/6-31+G* & -0.60 & -0.64 & -0.62 & -0.53 & -0.66 & -0.73 & -0.58 \\
B3LYP/6-311+G(2d,p) & -0.54 & -0.58 & & -0.47 & -0.59 & & \\
\hline
\end{tabular}

a) VEA in $\mathrm{eV}$ 
Table S5. Energies of the donors and its oxydized forms in solution in a. u. ${ }^{\text {a }}$

\begin{tabular}{|c|c|c|c|}
\hline \multicolumn{2}{|l|}{$\mathrm{RNu}=$} & $\begin{array}{c}\text { Solution phase energy of : } \\
\mathrm{RNu} \cdot-\end{array}$ & RNu \\
\hline & (7) & -1152.018892 & -1151.954468 \\
\hline & (8) & -1077.982134 & -1077.924567 \\
\hline & (9) & -1194.714586 & -1194.658098 \\
\hline & (10) & -1268.749451 & -1268.686653 \\
\hline $\mathrm{Me}_{3} \mathrm{Sn}$ & (11) & -587.252096 & -587.194717 \\
\hline
\end{tabular}

a) For calculations involving $\mathrm{Sn}$, the $\mathrm{LACVP}+^{*}$ basis has been used $\left(\mathrm{H}\right.$ to $\mathrm{Ar}$ elements use the same $6-31+\mathrm{G}^{*}$ basis and for $\mathrm{Sn}$, the $6-31+G^{*}$ plus the pseudopotential from Hay, P. J.; Wadt, W. R. J. Chem. Phys. 1985, 82, 299.)

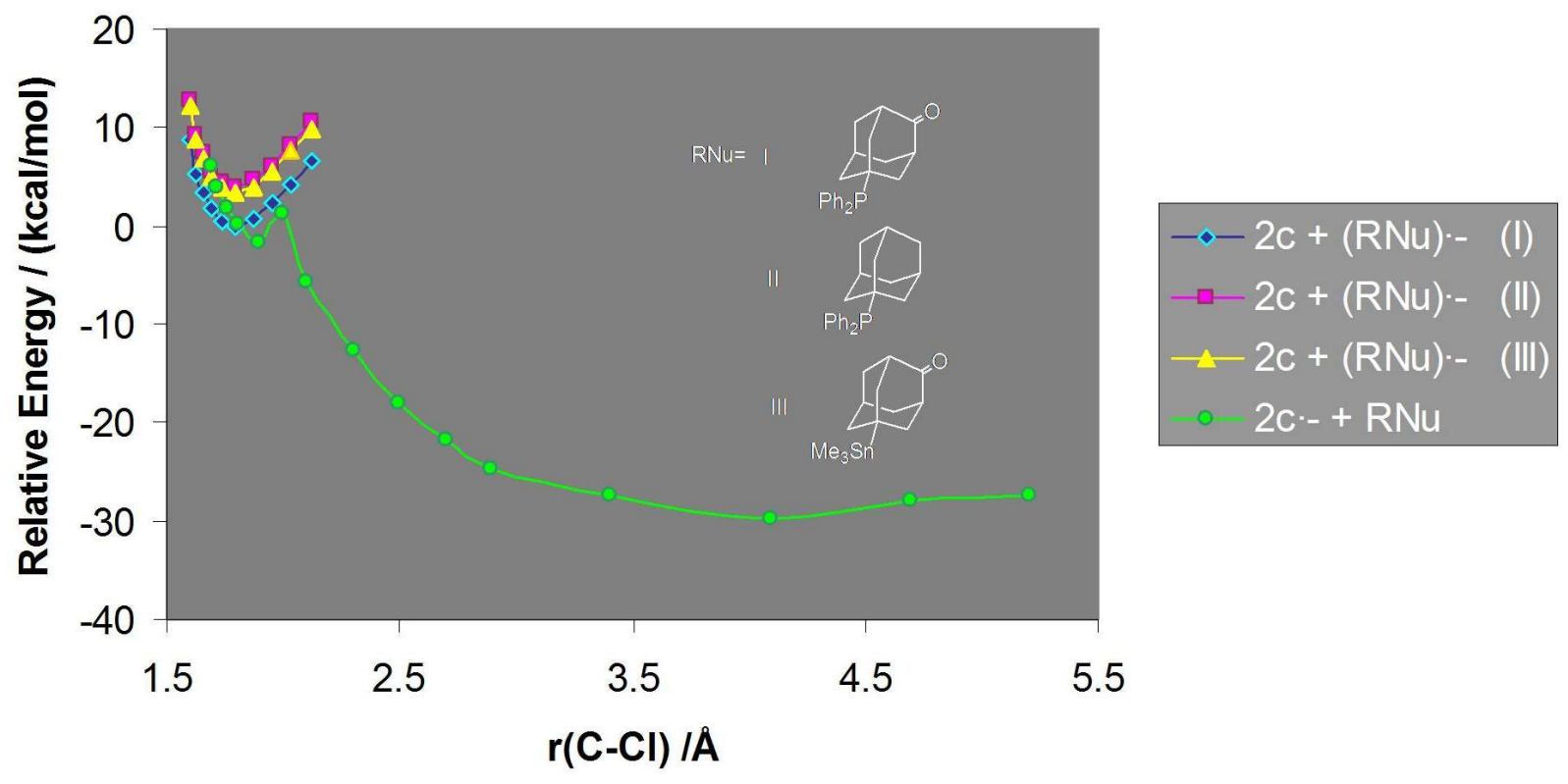

Figure_S 3. Same as main text figure 4, comparing the driving force given by three RNu-donors. 
NBO analysis has been performed on all solution phase stationary points informed in Table 1 (main text) as another standard ${ }^{17}$ population analysis in order to obtain quantitative properties useful to describe bonding and charge and spin distribution (for example spin population on radical center and hybridization scheme informed on Table 1). On the other hand, NBO analysis has also been applied as a qualitative tool in order to rationalize the relative importance of the bridge elements in allowing for the coupling between the $\pi(\mathrm{C}=\mathrm{O})$ and $\sigma *(\mathrm{C}-\mathrm{Cl})$ subsystems involved in the dissociative intra-ET. ${ }^{5,16}$ For this purpose, single point UHF/6$31 \mathrm{G}^{*}$ energy calculations have been done on the structures of the radical anions of $\mathbf{1 c}, \mathbf{2 c}$ and $\mathbf{1 b}$ (for $\mathbf{1 b}$, which does not reach a RA minimum, the structure of the shoulder (Fig. S1) at a fixed C-Cl length was used). The actual radical anion (i.e. unaltered Fock matrix) has been compared to different models in which specific interactions between natural bonding orbitals with high and low occupancy have been suppressed by setting their non-diagonal Fock matrix elements (in NBO basis) to zero; in NBO terminology, the comparison of the system before and after specific deletions.

The actual RA has been compared to four models in each case, which have been setup by deleting:

I. The direct, through space interaction, i.e. setting to zero the non-diagonal elements involving the bonding, non-bonding and antibonding $\pi$ orbitals with the antibonding and bonding $\sigma$ orbitals of the $\mathrm{C}-\mathrm{Cl}$.

II. the interaction through the shortest bridge, or the path involving the minimal number of C-C bonds (1 2 and 3 for the $\alpha, \beta$, and $\gamma$ oxo-subsituted species $\mathbf{1 a}, \mathbf{1 b}$, and $\mathbf{2 c}$, respectively). In the case of $\mathbf{2 c}$, there are two possible ways; for simplicity, only one of them is considered.

III. through the whole bridge (as II but decoupling all the possible paths)

IV. through the bridge and through space (I + III).

The actual systems have been compared in each case to the models (I-IV) in terms of the change in the canonical SOMO and LUMO orbitals, the spin population of the carbonyl carbon and the natural charge of the carbonyl oxygen (which are more localized on these center as well as the coupling $\pi$-bridge- $\sigma$ coupling is less effective). The results are summarized on Fig. S4. 

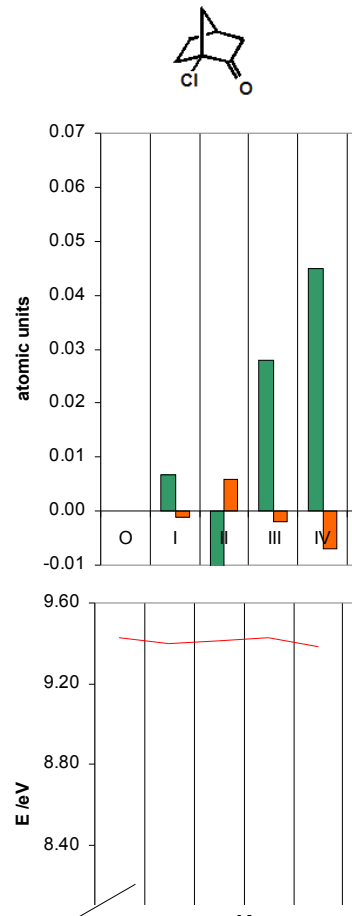

$1 b$

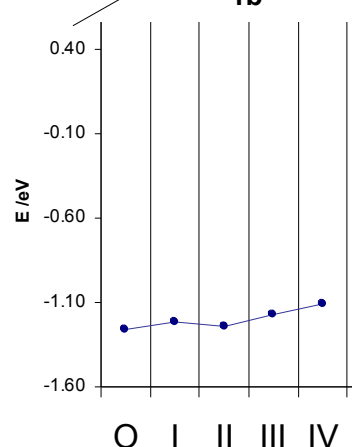

$b^{\circ}$
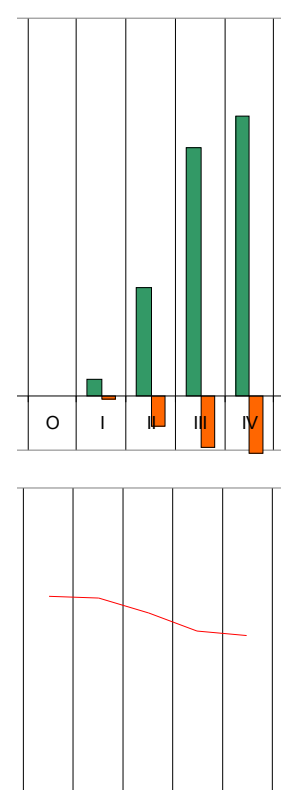

1c

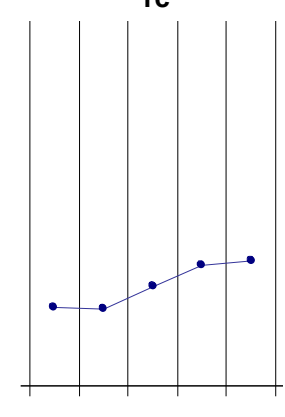

O I II III IV
D

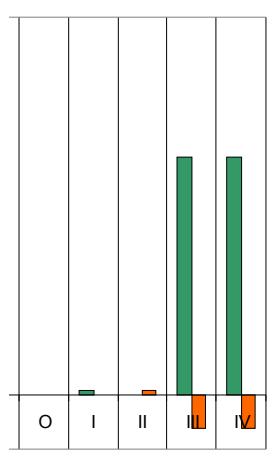

$\Delta q(\mathrm{O})$

$\Delta \operatorname{spin}(\mathrm{C})$
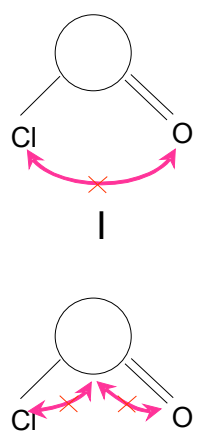

II

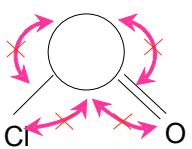

III

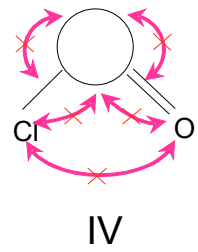

Figure S4. Summary of NBO results for models I-IV.

There are no important differences between the actual system and model I, except for the case of $\mathbf{1 b}$, which implies that the direct through space coupling only plays an appreciable role in the case of $\alpha$ oxo substituted species.

The effect of deletions II is by far smaller than III. This indicates that the polycycle skeleton as a whole (all possible ways, and the orientation of each sub-bridge, which is related to the structure and strain of the polycycle) determines the overall through-bond coupling, besides the number of $\mathrm{CC}$ bond separating the substituents. The magnitude of the overall effect is not essentially different for the different bridges tested. 